\title{
High-resolution spectroscopic observations of two chemically peculiar metal-poor stars: HD 10613 and $B D+04^{\circ} 2466^{\star}$
}

\author{
C. B. Pereira ${ }^{1}$ and N. A. Drake ${ }^{2}$ \\ ${ }^{1}$ Observatório Nacional, Rua José Cristino, 77. CEP 20921-400, São Cristóvão, Rio de Janeiro-RJ, Brazil \\ e-mail: claudio@on.br \\ 2 Sobolev Astronomical Institute, St. Petersburg State University, Universitetski pr. 28, St. Petersburg 198504, Russia
}

Received 22 August 2008 / Accepted 11 January 2009

\section{ABSTRACT}

\begin{abstract}
Aims. We determined the atmospheric parameters and abundance pattern of two chemically peculiar metal-poor stars: HD 10613 and $\mathrm{BD}+04^{\circ} 2466$ in order to better understand their evolutionary state and the nature of the s-element enhancement of these stars. Methods. We used high resolution optical spectroscopy. Atmospheric parameters and abundances were determined in the localthermodynamic-equilibrium model atmospheres of Kurucz using the spectral analysis code MOOG.

Results. We conclude that HD 10613 is another metal-poor barium star with $\mathrm{C} / \mathrm{O}=0.52$ and $[\mathrm{Fe} / \mathrm{H}]=-0.82$, while $\mathrm{BD}+04^{\circ} 2466$ is a $\mathrm{CH}$ star with $\mathrm{C} / \mathrm{O}=3.6$ and $[\mathrm{Fe} / \mathrm{H}]=-1.92$ rather than a metal-deficient barium star as it was previously classified. $\mathrm{BD}+04^{\circ} 2466$ appears to be enriched in lead with $[\mathrm{Pb} / \mathrm{Ce}]=+0.85$ and $[\mathrm{Pb} / \mathrm{La}]=+0.72$. For $\mathrm{BD}+04^{\circ} 2466$ the abundance of lead is in agreement with predictions from AGB models. Due to the low luminosity of these two stars, their observed s-process overabundance is better explained by mass-transfer in the past from an AGB star.
\end{abstract}

Key words. stars: abundances - stars: chemically peculiar - stars: binaries: general

\section{Introduction}

Barium and $\mathrm{CH}$ stars belong to a class of chemically peculiar stars where binarity is an essential requirement to explain their overabundance of carbon and the elements heavier than iron, such as barium. Barium and strontium are synthesized by neutron-capture reactions provided that the rate of neutron capture is slow compared to the beta-decay time scale of the radioactive nuclei involved in the chain. This nucleosynthesis process was called the s-process by Burbidge et al. (1957). The main site for this process is inside a star during its evolution through the AGB phase where the star develops helium burning thermal pulses (TP-AGB phase). A by-product of the thermal pulse is the production of both carbon and neutron-rich isotopes of heavy elements. Because of the deep convection zone of an AGB star, these nuclei are brought to the star's surface by the "third dredge up". However, both barium stars and CH stars are not luminous enough to be considered AGB stars having undergone a third dredge-up. Therefore, their overabundances of carbon and s-process elements are explained by mass-transfer in a binary system from a former AGB star (now a white dwarf in the system).

Although both barium stars and $\mathrm{CH}$ stars are formed by the same phenomena, they do not share the same properties. It seems that barium stars have orbital periods longer than $\mathrm{CH}$ stars and their eccentricities are greater (Vanture 1992a). The abundance pattern of barium stars and $\mathrm{CH}$ stars, as far as the observed overabundances of the elements created by the s-process are concerned, is similar, except for the $\mathrm{C} / \mathrm{O}$ ratio. $\mathrm{CH}$ stars present strong $\mathrm{C}_{2}$ bands and the $\mathrm{C} / \mathrm{O}$ ratio already determined so far is greater than unity (Vanture 1992b; this work) while for barium

\footnotetext{
* Based on observations made with the $1.52 \mathrm{~m}$ and $2.2 \mathrm{~m}$ telescope at the European Southern Observatory (La Silla, Chile).
}

stars the C/O ratio is less than unity (Barbuy et al. 1992; Allen \& Barbuy 2006; Drake \& Pereira 2008; this work).

Regarding the stellar population type, $\mathrm{CH}$ stars are clearly members of the halo population, they have high radial velocities and are metal-poor objects (Hartwick \& Cowley 1985). CH stars have been regarded as population II counterparts of the barium stars (Smith et al. 1996). Population studies done for barium stars show that they differ from the $\mathrm{CH}$ stars with respect to their distribution in the Galaxy. Barium stars are found in the disk and in the halo of the Galaxy (Gómez et al. 1997; Mennessier et al. 1997). These two studies show that barium stars can also be divided into groups according to their luminosities, kinematical and spatial parameters $\left(U_{0}, V_{0}, W_{0}\right.$ velocities and dispersion velocities and scale heights). These two studies also show that barium stars in the halo are very rare. Table 4 of Mennessier et al. (1997) shows that the number of barium star candidates in the halo (flagged by "H") comprises only $6 \%$ of the total sample investigated by these authors.

In 1991, Luck \& Bond (1991) (hereafter LB91) singled out a small class of four objects that they called "metal-deficient barium stars". They noticed that the stars of this group have a very strong $\mathrm{CH}$ band and very weak metallic lines but the strength of the $\mathrm{C}_{2}$ band is very weak, and because of that they could not be categorized as classical $\mathrm{CH}$ stars. These metal-deficient barium stars, according to LB91, would be the Population II analogs of classical barium stars.

In this work we continue our investigation of these "metaldeficient barium stars" not only from the LB91 sample but also searching for possible candidates in the literature. In a previous search we identified and analyzed HD 206983 (Junqueira \& Pereira 2001; Drake \& Pereira 2008). We now analyze one star that was suspected to be a metal-poor barium star by Catchpole et al. (1977) and also classified as a member of the halo 


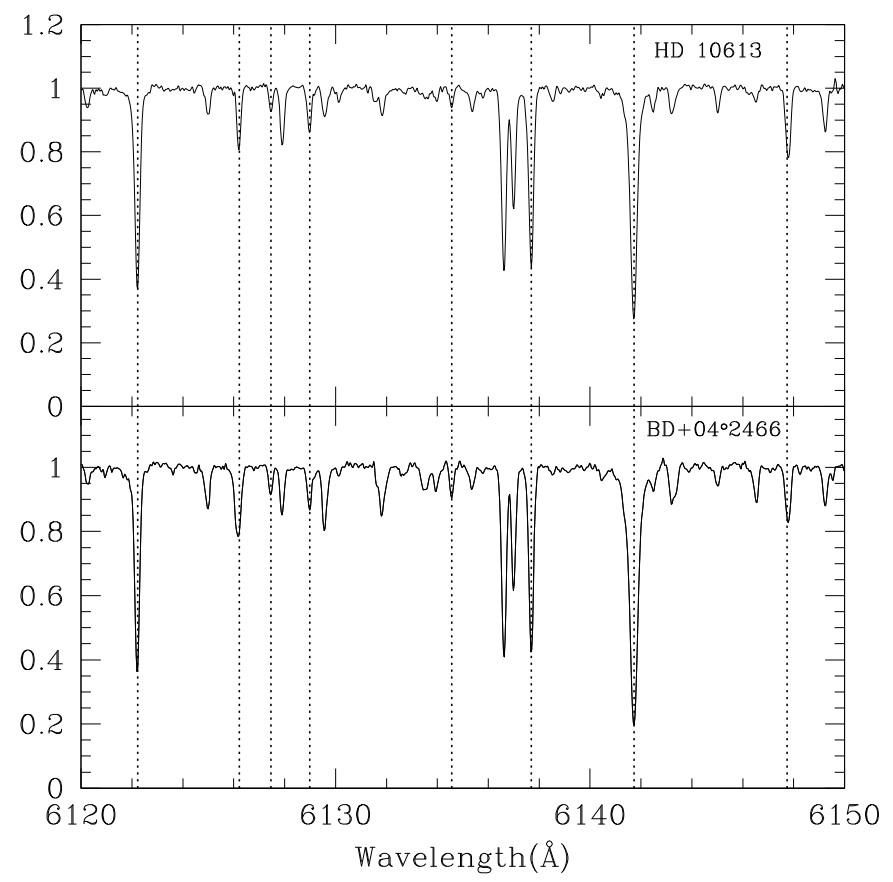

Fig. 1. Sample spectra of the stars analyzed in this work. Dotted vertical lines show the transitions of CaI 6122.23, TiI 6126.22, Zri 6127.48, Ni I 6128.98, Zri 6134.57, Fe I 6137.70, Ba II 6141.73, and Fe II 6147.83.

population by Goméz et al. (1997) and Mennesier et al. (1997), HD 10613. In addition we analyze $\mathrm{BD}+04^{\circ} 2466$ which belongs to this small sample of "metal-deficient barium stars" of LB91.

\section{Observations}

The high-resolution spectra of HD 10613 and BD+04²466 analyzed in this work were obtained with the FEROS (Fiberfed Extended Range Optical Spectrograph) echelle spectrograph (Kaufer et al. 1999) at the $1.52 \mathrm{~m}$ and $2.2 \mathrm{~m}$ ESO telescopes at La Silla (Chile), respectively, on the nights of November 25, 2001 (HD 10613) for $3600 \mathrm{~s}$ and April 3, 2007 (BD+04 ${ }^{\circ} 2466$ ) for $1200 \mathrm{~s}$. The FEROS spectral resolving power is $R=48000$, corresponding to 2.2 pixels of $15 \mu \mathrm{m}$, and the wavelength coverage goes from $3800 \AA$ to $9200 \AA$. The nominal $S / N$ ratio was evaluated by measuring the rms flux fluctuation in selected continuum windows, and the typical values were $S / N=100-150$ for both stars. The spectra were reduced with the MIDAS pipeline reduction package consisting of the following standard steps: CCD bias correction, flat-fielding, spectrum extraction, wavelength calibration, correction of barycentric velocity, and spectrum rectification. Figure 1 shows sample spectra of the programme stars in the 6120-6150 ^ region.

\section{Analysis and results}

\subsection{Line selection, measurements and oscillator strengths}

The atomic absorption lines selected in this study are basically the same as used in previous studies dedicated to the analysis of photospheric abundances of symbiotic stars (Pereira et al. 1998) and barium stars (Junqueira \& Pereira 2001; Pereira \& Junqueira 2003; Pereira 2005). The lines chosen are sufficiently unblended to yield reliable abundances. Table 1 shows the Fe I and Fe II lines employed in the analysis and also the lower
Table 1. Observed Fe I and Fe II lines.

\begin{tabular}{|c|c|c|c|c|c|}
\hline \multirow[b]{3}{*}{ Element } & \multirow[b]{3}{*}{$\lambda(\AA)$} & \multirow[b]{3}{*}{$\chi(\mathrm{eV})$} & \multirow[b]{3}{*}{$\log g f$} & \multicolumn{2}{|c|}{ Equivalent widths (mÅ) } \\
\hline & & & & HD & $\mathrm{BD}+04^{\circ}$ \\
\hline & & & & 10613 & 2466 \\
\hline \multirow[t]{64}{*}{$\mathrm{Fe} \mathrm{I}$} & 5022.24 & 3.98 & -0.490 & 93 & - \\
\hline & 5029.62 & 3.42 & -1.900 & 46 & - \\
\hline & 5044.21 & 2.85 & -2.040 & 73 & - \\
\hline & 5074.75 & 4.22 & -0.160 & 95 & - \\
\hline & 5083.34 & 0.96 & -2.910 & 127 & - \\
\hline & 5090.77 & 4.26 & -0.360 & 91 & - \\
\hline & 5150.84 & 0.99 & -3.000 & 136 & - \\
\hline & 5159.06 & 4.28 & -0.650 & 60 & - \\
\hline & 5171.59 & 1.49 & -1.760 & - & 100 \\
\hline & 5180.06 & 4.47 & -1.110 & 40 & - \\
\hline & 5194.94 & 1.56 & -2.090 & - & 82 \\
\hline & 5198.71 & 2.22 & -2.140 & 101 & 40 \\
\hline & 5202.34 & 2.18 & -1.840 & - & 65 \\
\hline & 5216.27 & 1.61 & -2.120 & 136 & - \\
\hline & 5232.94 & 2.94 & -0.080 & - & 100 \\
\hline & 5242.49 & 3.63 & -0.970 & 86 & 100 \\
\hline & 5250.21 & 0.12 & -4.920 & - & 23 \\
\hline & 5253.03 & 2.28 & -3.790 & 25 & - \\
\hline & 5281.79 & 3.04 & -0.830 & 117 & 57 \\
\hline & 5288.52 & 3.69 & -1.510 & 46 & - \\
\hline & 5302.31 & 3.28 & -0.740 & 114 & 56 \\
\hline & 5307.36 & 1.61 & -2.970 & 105 & 35 \\
\hline & 5321.11 & 4.43 & -1.190 & 38 & - \\
\hline & 5322.04 & 2.28 & -2.840 & 72 & 13 \\
\hline & 5339.93 & 3.27 & -0.680 & 122 & 57 \\
\hline & 5364.87 & 4.45 & 0.230 & 96 & 39 \\
\hline & 5369.96 & 4.37 & 0.540 & - & 52 \\
\hline & 5371.49 & 0.96 & -1.650 & - & 133 \\
\hline & 5373.71 & 4.47 & -0.710 & 47 & - \\
\hline & 5400.50 & 4.37 & -0.100 & 100 & 24 \\
\hline & 5405.77 & 0.99 & -1.850 & - & 120 \\
\hline & 5417.03 & 4.42 & -1.530 & 22 & - \\
\hline & 5424.07 & 4.32 & 0.580 & 128 & - \\
\hline & 5434.52 & 1.01 & -2.120 & - & 106 \\
\hline & 5445.04 & 4.39 & 0.040 & 91 & 38 \\
\hline & 5466.40 & 4.37 & -0.570 & 62 & - \\
\hline & 5506.78 & 0.99 & -2.800 & - & 78 \\
\hline & 5554.90 & 4.55 & -0.380 & 73 & - \\
\hline & 5567.39 & 2.61 & -2.560 & 74 & - \\
\hline & 5569.62 & 3.42 & -0.490 & 135 & 63 \\
\hline & 5576.09 & 3.43 & -0.850 & 96 & - \\
\hline & 5638.26 & 4.22 & -0.720 & 67 & - \\
\hline & 5658.82 & 3.40 & -0.810 & - & 47 \\
\hline & 5679.02 & 4.65 & -0.770 & 39 & - \\
\hline & 5691.50 & 4.30 & -1.370 & 32 & - \\
\hline & 5731.76 & 4.26 & -1.150 & 45 & - \\
\hline & 5762.99 & 4.21 & -0.410 & 83 & 25 \\
\hline & 5806.73 & 4.61 & -0.900 & 34 & - \\
\hline & 5814.81 & 4.28 & -1.820 & 13 & - \\
\hline & 5852.22 & 4.55 & -1.180 & 25 & - \\
\hline & 5934.65 & 3.93 & -1.020 & 60 & - \\
\hline & 6020.17 & 4.61 & -0.210 & - & 21 \\
\hline & 6024.06 & 4.55 & -0.060 & 81 & 27 \\
\hline & 6027.05 & 4.08 & -1.090 & 52 & - \\
\hline & 6056.01 & 4.73 & -0.400 & 49 & - \\
\hline & 6065.48 & 2.61 & -1.530 & 124 & 56 \\
\hline & 6082.71 & 2.22 & -3.580 & 42 & - \\
\hline & 6093.64 & 4.61 & -1.350 & 14 & - \\
\hline & 6096.66 & 3.98 & -1.780 & 25 & - \\
\hline & 6136.61 & 2.45 & -1.400 & 134 & 74 \\
\hline & 6137.69 & 2.59 & -1.400 & 131 & 62 \\
\hline & 6151.62 & 2.18 & -3.290 & 57 & - \\
\hline & 6165.36 & 4.14 & -1.470 & 32 & - \\
\hline & 6173.34 & 2.22 & -2.880 & 85 & - \\
\hline
\end{tabular}


Table 1. continued.

\begin{tabular}{|c|c|c|c|c|c|}
\hline \multirow[b]{3}{*}{ Element } & \multirow[b]{3}{*}{$\lambda(\AA)$} & \multirow[b]{3}{*}{$\chi(\mathrm{eV})$} & \multirow[b]{3}{*}{$\log g f$} & \multicolumn{2}{|c|}{ Equivalent widths $(\mathrm{m} \AA$ ) } \\
\hline & & & & HD & $\mathrm{BD}+04^{\circ}$ \\
\hline & & & & 10613 & 2466 \\
\hline & 6187.99 & 3.94 & -1.570 & 32 & - \\
\hline & 6191.56 & 2.43 & -1.420 & - & 70 \\
\hline & 6200.32 & 2.60 & -2.440 & 76 & 16 \\
\hline & 6213.43 & 2.22 & -2.480 & 95 & 25 \\
\hline & 6252.56 & 2.40 & -1.720 & 124 & 57 \\
\hline & 6254.26 & 2.28 & -2.440 & - & 32 \\
\hline & 6265.13 & 2.18 & -2.550 & 98 & 31 \\
\hline & 6322.69 & 2.59 & -2.430 & 72 & - \\
\hline & 6380.74 & 4.19 & -1.320 & 40 & - \\
\hline & 6393.60 & 2.43 & -1.430 & 130 & 67 \\
\hline & 6411.65 & 3.65 & -0.660 & 110 & 40 \\
\hline & 6419.95 & 4.73 & -0.090 & 63 & - \\
\hline & 6421.35 & 2.28 & -2.010 & 123 & 51 \\
\hline & 6430.85 & 2.18 & -2.010 & 125 & 57 \\
\hline & 6592.91 & 2.72 & -1.470 & 111 & - \\
\hline & 6593.87 & 2.44 & -2.420 & 96 & - \\
\hline & 6609.11 & 2.56 & -2.690 & 71 & - \\
\hline & 6752.71 & 4.64 & -1.200 & 21 & - \\
\hline & 6810.26 & 4.61 & -1.200 & 28 & - \\
\hline & 6820.37 & 4.64 & -1.170 & 23 & - \\
\hline & 6858.15 & 4.61 & -0.930 & 39 & - \\
\hline \multirow[t]{14}{*}{ Fe II } & 4993.35 & 2.81 & -3.670 & 35 & 15 \\
\hline & 5197.56 & 3.23 & -2.250 & - & 59 \\
\hline & 5234.62 & 3.22 & -2.240 & - & 67 \\
\hline & 5276.00 & 3.20 & -1.910 & - & 78 \\
\hline & 5284.10 & 2.89 & -3.010 & - & 34 \\
\hline & 5325.56 & 3.22 & -3.170 & 35 & 18 \\
\hline & 5425.25 & 3.20 & -3.210 & 38 & 18 \\
\hline & 5534.83 & 3.25 & -2.770 & 58 & - \\
\hline & 5991.37 & 3.15 & -3.560 & 25 & - \\
\hline & 6149.25 & 3.89 & -2.720 & 26 & - \\
\hline & 6247.55 & 3.89 & -2.340 & 43 & 25 \\
\hline & 6369.46 & 2.89 & -4.190 & 15 & - \\
\hline & 6416.92 & 3.89 & -2.680 & 28 & - \\
\hline & 6432.68 & 2.89 & -3.580 & 36 & - \\
\hline
\end{tabular}

excitation potential, $\chi$, of the transitions, the $\log g f$ values, and the measured equivalent widths. The latter were obtained by fitting Gaussian profiles to the observed ones. The $\log g f$ values for the Fe I and Fe II lines given in Table 1 were taken from Lambert et al. (1996).

\subsection{Determination of the atmospheric parameters}

The determination of stellar atmospheric parameters, effective temperature $\left(T_{\text {eff }}\right)$, surface gravity $(\log g)$, microturbulence $(\xi)$ and metallicity $[\mathrm{Fe} / \mathrm{H}]$ (throughout this paper, we use the notation $\left.[\mathrm{X} / \mathrm{H}]=\log \left(N_{\mathrm{X}} / N_{\mathrm{H}}\right)_{\star}-\log \left(N_{\mathrm{X}} / N_{\mathrm{H}}\right)_{\odot}\right)$ is prerequisite for a determination of photospheric abundance. The gravity was determined by forcing the Fe I and Fe II lines to yield the same iron abundance at the selected effective temperature. The microturbulent velocity was determined by forcing the abundance determined from individual Fe I lines to show no dependence on equivalent width. The effective temperature was derived by requiring that the abundances calculated for the $\mathrm{Fe} I$ lines do not show any dependence upon excitation potential. The solution thus found is unique, depending only on a set of Fe I, II lines and the employed atmospheric model, and yields as a byproduct the metallicity of the star $([\mathrm{Fe} / \mathrm{H}])$. The atmospheric parameters were determined using the local thermodynamic equilibrium (LTE) atmosphere models of Kurucz (1993) and the
Table 2. Atmospheric parameters, radial velocities and galactic latitudes of HD 10613 and BD $+04^{\circ} 2466$.

\begin{tabular}{lccc}
\hline \hline & HD 10613 & ${\mathrm{BD}+04^{\circ} 2466^{a}}$ & ${\mathrm{BD}+04^{\circ} 2466^{b}}^{\circ}$ \\
\hline$T_{\text {eff }}(\mathrm{K})$ & $5100 \pm 120$ & $5100 \pm 100$ & 4800 \\
$\log g(\mathrm{dex})$ & $2.8 \pm 0.1$ & $1.8 \pm 0.2$ & 1.8 \\
{$[\mathrm{Fe} / \mathrm{H}](\mathrm{dex})$} & $-0.82 \pm 0.1$ & $-1.92 \pm 0.08$ & -1.85 \\
$\xi\left(\mathrm{km} \mathrm{s}^{-1}\right)$ & $1.6 \pm 0.2$ & $1.6 \pm 0.2$ & 1.8 \\
$V_{r}\left(\mathrm{~km} \mathrm{~s}^{-1}\right)$ & $+89.3 \pm 0.43$ & $+38.5 \pm 0.50$ & - \\
gal. latitude $\left(b^{\circ}\right)$ & -74.7 & +39.2 & - \\
\hline
\end{tabular}

${ }^{a}$ This work; ${ }^{b}$ LB91.

spectral analysis code MOOG (Sneden 1973). The final adopted atmospheric parameters are given in Table 2. This table also shows the radial velocities of the stars as well as their galactic latitudes. Figure 2 shows abundances of the individual Fe I lines plotted versus the lower excitation potential and the reduced line strength, respectively, for the stellar parameters adopted for HD 10613 and BD+04 2466.

The internal errors in the adopted effective temperature $\left(T_{\text {eff }}\right)$ and microturbulent velocity $(\xi)$ can be determined from the uncertainty in the slope of the Fe I abundance versus excitation potential and Fe I abundance versus reduced equivalent width $\left(W_{\lambda} / \lambda\right)$ relations. These quantities are given in Table 2 . The standard deviation in $\log g$ was set by changing this parameter around the adopted solution until the Fe I and Fe II mean abundances differ by exactly one standard deviation of the $[\mathrm{Fe} \mathrm{I} / \mathrm{H}]$ mean value.

\subsection{Abundance analysis}

The abundances of chemical elements were determined with the local thermodynamic equilibrium (LTE) model-atmosphere techniques described in above section. In brief, equivalent widths were calculated by integration through a model atmosphere and are compared with the observed equivalent widths. The calculation is repeated, changing the abundance of the element in question, until a match is achieved. The line-synthesis code MOOG was used to carry out the calculations.

Table 3 shows the atomic lines used to derive the abundances of the elements and Table 4 provides a list of the number of lines employed (or number of spectral regions in the case of carbon and nitrogen whose abundances were determined using molecular lines) for each species, $n$, and the standard deviation as well as the $\mathrm{C} / \mathrm{O}$ ratio.

The abundance of manganese is based on the MnI lines $\lambda 6013$ and $\lambda 6021 \AA$ for HD 10613 and only $\lambda 6021 \AA$ for $\mathrm{BD}+04^{\circ} 2466$. The europium abundance for HD 10613 is based on the Eu II line $\lambda 6645 \AA$ and the abundance of copper is based on the $\mathrm{Cu}$ I line $\lambda 5105 \AA$. The abundances for these elements were obtained through the spectrum synthesis technique. The synthetic spectra were broadened to match the instrumental profile. All fine-structure and hyperfine-structure components for the lines of $\mathrm{Cu}$ I, MnI and Eu II were explicitly included. The $g f$-values for $\mathrm{Cu}$ I are from Koch \& Richter (1968), those for Mn I and Eu II are from del Peloso et al. (2005).

Carbon, nitrogen and oxygen abundances as well as the ${ }^{12} \mathrm{C} /{ }^{13} \mathrm{C}$ isotopic ratio were also determined with spectrum synthesis technique in the local thermodynamic equilibrium (LTE) atmosphere models of Kurucz (1993). Since the abundances of the $\mathrm{CNO}$ elements are interdependent because of the association of carbon and oxygen into CO molecules in the atmospheres of 

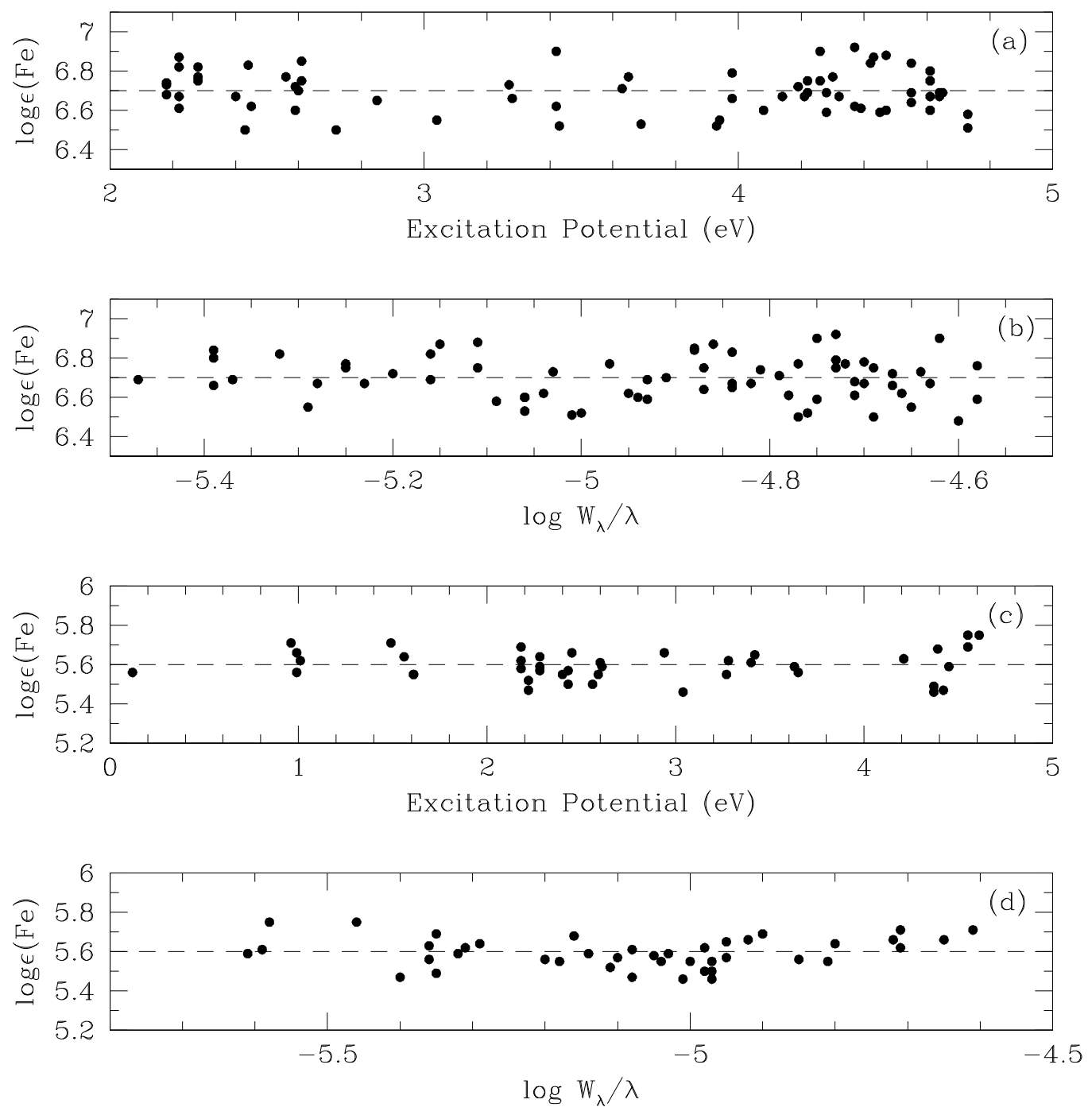

Fig. 2. The iron abundance derived from individual Fe I lines, $\log \varepsilon(\mathrm{Fe})$, versus excitation potential a) and versus the reduced equivalent width, $\log \left(W_{\lambda} / \lambda\right)$, diagram b), for HD 10613 and the same diagrams $(\mathbf{c})$ and d)) for BD+04 2466 . For HD 10613, 81 lines of Fe I,II were employed in the determination of $T_{\text {eff }}, \log g, \xi$, and $\log \varepsilon(\mathrm{Fe})$. The calculation was performed with the adopted model $T_{\text {eff }}=5100 \mathrm{~K}, \log g=2.8, \log \varepsilon(\mathrm{Fe})=$ 6.7 and $\xi=1.6 \mathrm{~km} \mathrm{~s}^{-1}$. For BD+04 2466,50 lines of Fe I,II were employed in the determination of $T_{\text {eff }}, \log g$, $\xi$, and $\log \varepsilon(\mathrm{Fe})$. The calculation was done with the adopted model $T_{\text {eff }}=5100 \mathrm{~K}, \log g=1.8, \log \varepsilon(\mathrm{Fe})=5.6$ and $\xi=1.6 \mathrm{~km} \mathrm{~s}^{-1}$. The absence of any trend of the individual Fe abundances with excitation potential and microturbulence shows that correct values for $T_{\text {eff }}$ and microturbulence velocity were chosen.

cool giants, we carried out an iterative procedure of CNO abundance determinations until all abundances of these three elements agreed.

The carbon abundances were derived using different spectral regions:

a) $\mathrm{C}_{2}(0,1)$ band head of the Swan system $A^{3} \Pi_{g}-X^{3} \Pi_{u}$ at $5635 \AA$. The electron oscillator strength, $f_{\mathrm{el}}=0.033$, was taken from Lambert (1978). The Hönl-London factors for the rotational lines were calculated using the formula from Kovacs (1969). Franck-Condon factors were calculated according to Dwivedi et al. (1978). However, as was shown by our calculations, the $(0,1)$ band of the Swan system does not present a significant dependence of the Franck-Condon factors the value of $J$. Thus the value $q_{0,1}=0.2206$ was adopted for all rotational lines of the $(0,1)$ band. The small contribution of faint $\mathrm{CN}(5,0)$ and $(10,4)$ bands of the $\mathrm{CN}$ red system was taken into account. The $\mathrm{C}_{2}$ dissociation energy of $D_{0}\left(C_{2}\right)=6.15 \mathrm{eV}$ was adopted (Huber \& Herzberg 1979). The wavelengths of the $\mathrm{C}_{2}$ features of the $(0,1)$ band were taken from Phillips \& Davis (1968). The observed and synthetic spectra of both stars of our sample in the region around $5635 \AA$ are shown in Fig. 3 .

b) $\mathrm{C}_{2}$ feature at $5086 \AA$. The oscillator strengths and wavelengths of $\mathrm{C}_{2}$ lines were taken from Lambert \& Ries (1981).

c) $\mathrm{CH}$ lines of the $A^{2} \Delta-X^{2} \Pi$ system. The oscillator strengths and wavelengths of the ${ }^{12} \mathrm{CH}$ and ${ }^{13} \mathrm{CH}$ lines were taken from the SCAN database (Jørgensen et al. 1996). The CH dissociation energy is well determined and is equal to $D_{0}(\mathrm{CH})=$ $3.45 \mathrm{eV}$ (Lambert 1978).

The nitrogen abundances were obtained by comparing the observed and theoretical line profiles for the ${ }^{12} \mathrm{CN}$ lines of the $(2,0)$ band of the $\mathrm{CN}$ red system $A^{2} \Pi-X^{2} \Sigma$ in the 7994-8020 $\AA$ wavelength range. The oscillator strength of the $(0,2)$ band of $f_{2,0}=8.4 \times 10^{-4}$ (Sneden \& Lambert 1982) was used. Hönl-London factors were calculated using the Schadee (1964) formula. The dissociation energy $D_{0}(\mathrm{CN})=7.65 \mathrm{eV}$ (Bauschlicher et al. 1988; Lambert 1994) was used. The wavelengths of the ${ }^{12} \mathrm{CN}$ lines were taken from 
Table 3. Other lines studied.

\begin{tabular}{|c|c|c|c|c|c|c|}
\hline \multirow[b]{3}{*}{$\lambda(\AA)$} & \multirow[b]{3}{*}{ Species } & \multirow[b]{3}{*}{$\chi(\mathrm{eV})$} & \multirow[b]{3}{*}{$a f$} & \multirow[b]{3}{*}{ Ref } & \multicolumn{2}{|c|}{ Equivalent widths $(\mathrm{m} \AA)$} \\
\hline & & & & & HD & $\mathrm{BD}+04^{\circ}$ \\
\hline & & & & & 10613 & 2466 \\
\hline 5682.65 & $\mathrm{NaI}$ & 2.10 & $1.995 \mathrm{e}-01$ & PS & 50 & 10 \\
\hline 5688.22 & $\mathrm{NaI}$ & 2.10 & $3.981 \mathrm{e}-01$ & PS & 77 & 20 \\
\hline 8717.83 & $\mathrm{Mg}_{\mathrm{I}}$ & 5.91 & $1.956 \mathrm{e}-01$ & WSM & 37 & - \\
\hline 8736.04 & Mg I & 5.94 & $4.571 \mathrm{e}-01$ & WSM & 67 & - \\
\hline 5793.08 & Si I & 4.93 & $8.600 \mathrm{e}-03$ & R03 & 30 & 10 \\
\hline 6125.03 & Si I & 5.61 & $2.884 \mathrm{e}-02$ & E93 & 21 & - \\
\hline 6145.02 & Si I & 5.61 & $3.715 e-02$ & E93 & 18 & - \\
\hline 6155.14 & Si I & 5.62 & $1.698 \mathrm{e}-01$ & E93 & 47 & 13 \\
\hline 7800.00 & Si I & 6.18 & $1.905 \mathrm{e}-01$ & E93 & 24 & - \\
\hline 8742.45 & Si I & 5.87 & $3.090 \mathrm{e}-01$ & E93 & 63 & - \\
\hline 6102.73 & $\mathrm{CaI}$ & 1.88 & $1.621 \mathrm{e}-01$ & D2002 & 123 & 63 \\
\hline 6122.23 & $\mathrm{Ca} \mathrm{I}$ & 1.89 & $4.786 \mathrm{e}-01$ & D2002 & - & 95 \\
\hline 6161.30 & $\mathrm{Ca} \mathrm{I}$ & 2.52 & $5.370 \mathrm{e}-02$ & E93 & 71 & 16 \\
\hline 6162.18 & $\mathrm{CaI}$ & 1.90 & $8.128 \mathrm{e}-01$ & D2002 & - & 107 \\
\hline 6166.44 & $\mathrm{Ca} \mathrm{I}$ & 2.52 & $7.244 \mathrm{e}-02$ & R03 & 68 & 13 \\
\hline 6169.04 & $\mathrm{Ca} \mathrm{I}$ & 2.52 & $1.585 \mathrm{e}-01$ & R03 & 83 & 39 \\
\hline 6169.56 & $\mathrm{Ca} I$ & 2.53 & $3.311 \mathrm{e}-01$ & DS91 & 98 & 39 \\
\hline 6439.08 & $\mathrm{Ca} \mathrm{I}$ & 2.52 & $2.951 \mathrm{e}+00$ & D2002 & 147 & 92 \\
\hline 6455.60 & $\mathrm{CaI}$ & 2.51 & $5.129 \mathrm{e}-02$ & R03 & 57 & 33 \\
\hline 6471.66 & $\mathrm{Ca} I$ & 2.51 & $2.041 \mathrm{e}-01$ & S86 & 91 & 33 \\
\hline 6493.79 & $\mathrm{Ca} \mathrm{I}$ & 2.52 & $7.762 \mathrm{e}-01$ & DS91 & 120 & 60 \\
\hline 5239.82 & Sc II & 1.45 & $1.698 \mathrm{e}-01$ & MFW & 77 & 39 \\
\hline 5526.82 & Sc II & 1.77 & $1.318 \mathrm{e}+00$ & MFW & 93 & 58 \\
\hline 5657.88 & Sc II & 1.51 & $2.884 \mathrm{e}-01$ & GS & 78 & 43 \\
\hline 6245.62 & Sc II & 1.51 & $9.555 e-01$ & R03 & - & 15 \\
\hline 6604.60 & Sc II & 1.36 & $5.012 \mathrm{e}-02$ & R03 & 53 & 19 \\
\hline 4533.25 & Ti I & 0.85 & $3.388 \mathrm{e}+00$ & D2002 & - & 69 \\
\hline 4534.78 & Ti I & 0.84 & $1.905 e+00$ & D2002 & 111 & 57 \\
\hline 4981.72 & Ti I & 0.84 & $3.162 \mathrm{e}+00$ & D2002 & 139 & - \\
\hline 5087.06 & Ti I & 1.43 & $1.445 \mathrm{e}-01$ & E93 & 44 & - \\
\hline 5113.45 & Ti I & 1.44 & $1.318 \mathrm{e}-01$ & E93 & 35 & - \\
\hline 5866.46 & Ti I & 1.07 & $1.345 \mathrm{e}-01$ & E93 & 67 & - \\
\hline 6091.18 & Ti I & 2.27 & $4.266 \mathrm{e}-01$ & R03 & 64 & - \\
\hline 6126.22 & Ti I & 1.05 & $4.266 \mathrm{e}-02$ & $\mathrm{R} 03$ & 135 & - \\
\hline 6261.10 & Ti I & 1.43 & $3.311 \mathrm{e}-01$ & B86 & 66 & - \\
\hline 5296.70 & CrI & 0.98 & $4.074 \mathrm{e}-02$ & GS & 103 & 28 \\
\hline 5300.75 & CrI & 0.98 & $7.413 \mathrm{e}-03$ & GS & 66 & - \\
\hline 5345.81 & Cr I & 1.00 & $1.047 \mathrm{e}-01$ & GS & 140 & 50 \\
\hline 5348.33 & Cr I & 1.00 & $5.129 \mathrm{e}-02$ & GS & 109 & 28 \\
\hline 5409.80 & Cr I & 1.03 & $1.905 \mathrm{e}-01$ & GS & - & 60 \\
\hline 6330.09 & Cr I & 0.94 & $1.202 \mathrm{e} 093$ & R03 & 37 & - \\
\hline 5084.11 & $\mathrm{Ni} \mathrm{I}$ & 3.680 & $6.607 \mathrm{e}-01$ & E93 & 72 & - \\
\hline 5115.40 & Ni I & 3.834 & $5.248 \mathrm{e}-01$ & R03 & 55 & - \\
\hline 6176.82 & Ni I & 4.088 & $5.450 \mathrm{e}-01$ & R03 & 44 & - \\
\hline 6327.60 & Ni I & 1.68 & $7.709 \mathrm{e}-04$ & MFW & 46 & - \\
\hline 6482.80 & Ni I & 1.94 & $2.344 \mathrm{e}-02$ & MFW & 44 & - \\
\hline 6586.33 & $\mathrm{Ni} I$ & 1.95 & $1.549 \mathrm{e}-03$ & MFW & 39 & - \\
\hline 6643.64 & Ni I & 1.68 & $9.332 \mathrm{e}-03$ & MFW & 100 & 24 \\
\hline 6767.77 & Ni I & 1.83 & $6.761 \mathrm{e}-03$ & MFW & 88 & 23 \\
\hline 6772.32 & Ni I & 3.66 & $1.072 \mathrm{e}-01$ & R03 & 35 & 15 \\
\hline 7788.93 & Ni I & 1.95 & $1.023 \mathrm{e}-02$ & E93 & 102 & - \\
\hline 4722.16 & Zn I & 4.01 & $4.074 \mathrm{e}-01$ & BG80 & - & 40 \\
\hline 4810.53 & Zn I & 4.06 & $6.761 \mathrm{e}-01$ & BG80 & 66 & 35 \\
\hline 4883.68 & Y II & 1.08 & $1.175 \mathrm{e}+00$ & SN96 & - & 79 \\
\hline 5087.43 & Y II & 1.08 & $6.761 \mathrm{e}-01$ & SN96 & 92 & 57 \\
\hline 5200.41 & Y II & 0.99 & $2.692 \mathrm{e}-01$ & SN96 & 86 & 48 \\
\hline 5205.72 & $\mathrm{Y}_{\mathrm{II}}$ & 1.03 & $4.571 \mathrm{e}-01$ & SN96 & - & 62 \\
\hline 5289.81 & Y II & 1.03 & $1.412 \mathrm{e}-02$ & VWR & 33 & - \\
\hline 5402.78 & Y II & 1.84 & $3.631 \mathrm{e}-01$ & R03 & 49 & 15 \\
\hline 6127.46 & $\mathrm{ZrI}$ & 0.15 & $8.710 \mathrm{e}-02$ & H82 & 15 & - \\
\hline 6134.57 & $\mathrm{ZrI}$ & 0.00 & $5.248 \mathrm{e}-02$ & B81 & 15 & - \\
\hline 6143.18 & ZrI & 0.07 & $7.943 \mathrm{e}-02$ & B81 & 15 & - \\
\hline 4208.98 & Zr II & 0.71 & $3.467 \mathrm{e}-01$ & SN96 & - & 68 \\
\hline 4317.32 & ZrII & 0.71 & $4.169 \mathrm{e}-02$ & SN96 & 33 & 23 \\
\hline
\end{tabular}


Table 3. continued.

\begin{tabular}{|c|c|c|c|c|c|c|}
\hline \multirow[b]{3}{*}{$\lambda(\AA)$} & \multirow[b]{3}{*}{ Species } & \multirow[b]{3}{*}{$\chi(\mathrm{eV})$} & \multirow[b]{3}{*}{$g f$} & \multirow[b]{3}{*}{ Ref } & \multicolumn{2}{|c|}{ Equivalent widths $(\mathrm{m} \AA)$} \\
\hline & & & & & HD & $\mathrm{BD}+04^{\circ}$ \\
\hline & & & & & 10613 & 2466 \\
\hline 4816.50 & Zr II & 1.01 & $1.000 \mathrm{e}-02$ & VWR & 28 & 10 \\
\hline 5112.27 & Zr II & 1.66 & $1.738 \mathrm{e}-01$ & E93 & 49 & 19 \\
\hline 6496.90 & Ba II & 0.60 & $4.170 \mathrm{e}-01$ & WM80 & 220 & 176 \\
\hline 4934.83 & La II & 1.25 & $1.191 \mathrm{e}-01$ & VWR & 18 & - \\
\hline 5303.53 & La II & 0.32 & $4.467 \mathrm{e}-02$ & VWR & 50 & 23 \\
\hline 5880.63 & La II & 0.24 & $1.479 \mathrm{e}-02$ & R04 & 14 & - \\
\hline 6320.42 & La II & 0.17 & $3.030 \mathrm{e}-02$ & VWR & 60 & 22 \\
\hline 6390.48 & La II & 0.32 & $2.750 \mathrm{e}-02$ & S96 & 54 & 25 \\
\hline 6774.33 & La II & 0.12 & $1.778 \mathrm{e}-02$ & S96 & 52 & 20 \\
\hline 5117.17 & Ce II & 1.40 & $1.023 \mathrm{e}+00$ & VWR & 41 & - \\
\hline 5187.45 & Ce II & 1.21 & $1.995 \mathrm{e}+00$ & VWR & 55 & 30 \\
\hline 5274.24 & Ce II & 1.28 & $2.450 \mathrm{e}+00$ & VWR & 56 & 35 \\
\hline 5330.58 & Ce II & 0.87 & $7.383 \mathrm{e}-01$ & VWR & 44 & 24 \\
\hline 5472.30 & Ce II & 1.25 & $1.650 \mathrm{e}-01$ & VWR & 36 & 18 \\
\hline 6051.80 & Ce II & 0.23 & $2.512 \mathrm{e}-02$ & S96 & 25 & 10 \\
\hline 4811.34 & $\mathrm{Nd}$ II & 0.06 & $9.660 \mathrm{e}-02$ & VWR & 81 & 47 \\
\hline 4820.34 & $\mathrm{Nd}$ II & 0.20 & $6.902 \mathrm{e}-02$ & VWR & - & 37 \\
\hline 4959.12 & $\mathrm{Nd}$ II & 0.06 & $1.213 \mathrm{e}-01$ & VWR & 84 & 53 \\
\hline 4989.95 & $\mathrm{Nd}$ II & 0.63 & $2.377 \mathrm{e}-01$ & VWR & 85 & 40 \\
\hline 5063.72 & $\mathrm{Nd}$ II & 0.98 & $1.746 \mathrm{e}-01$ & VWR & 37 & 17 \\
\hline 5130.59 & $\mathrm{Nd}$ II & 1.30 & $1.259 \mathrm{e}+00$ & SN96 & 79 & 79 \\
\hline 5212.36 & $\mathrm{Nd}$ II & 0.20 & $1.995 \mathrm{e}-01$ & E93 & 71 & - \\
\hline 5249.58 & $\mathrm{Nd}$ II & 0.98 & $1.202 \mathrm{e}+00$ & SN96 & 74 & 45 \\
\hline 5311.46 & $\mathrm{Nd}$ II & 0.99 & $2.754 \mathrm{e}-01$ & SN96 & 47 & 16 \\
\hline 5319.81 & $\mathrm{Nd}$ II & 0.55 & $4.467 e-01$ & SN96 & 86 & 52 \\
\hline 5361.47 & $\mathrm{Nd}$ II & 0.68 & $3.981 \mathrm{e}-01$ & SN96 & 78 & 33 \\
\hline 5416.38 & $\mathrm{Nd}$ II & 0.86 & $1.047 \mathrm{e}-01$ & VWR & 31 & - \\
\hline 5431.54 & Nd II & 1.12 & $3.491 \mathrm{e}-01$ & VWR & 39 & - \\
\hline 5442.26 & Nd II & 0.68 & $1.259 \mathrm{e}-01$ & SN96 & 45 & 16 \\
\hline 5740.88 & Nd II & 1.16 & $2.754 \mathrm{e}-01$ & VWR & 32 & - \\
\hline 5842.39 & Nd II & 1.28 & $2.506 \mathrm{e}-01$ & VWR & 24 & - \\
\hline
\end{tabular}

References to Table 3: BG80: Biémont \& Godefroid (1980); B81: Biémont et al. (1981); D2002: Depagne et al. (2002); DS91: Drake \& Smith (1991); E93: Edvardsson et al. (1993); GS: Gratton \& Sneden (1988); H82: Hannaford et al. (1982); MFW: Martin et al. (1988); PS: Preston \& Sneden (2001); R03: Reddy et al. (2003); R04: Reyniers et al. (2004); S86: Smith et al. (1986); S96: Smith et al. (1996); SN96: Sneden et al. (1996); VWR: van Winckel \& Reyniers (2000); WSM: Wiese et al. (1969); WM80: Wiese \& Martin (1980).

Davis \& Phillips (1963) and those of ${ }^{13} \mathrm{CN}$ lines from Wyller (1966). Contamination of the $\mathrm{CN}$ features by the telluric $\mathrm{H}_{2} \mathrm{O}$ lines was eliminated by dividing our spectra by the high rotating hot star spectrum.

For the determination of the oxygen abundances we used the [O I] forbidden line at $6300.304 \AA$. In our calculations, we used for this line the transition probability $\log g f=-9.717 \mathrm{ob}-$ tained by Allende Prieto et al. (2001) in their analysis of solar oxygen abundance. This value is only slightly higher than the value of $\log g f=-9.75$ suggested by Lambert (1978). The [O I] $6300.304 \AA$ line is blended with the Ni I line at $6300.339 \AA$ which provides only a small contribution to the oxygen line. The oscillator strength of the Ni I line, $\log g f=-2.31$, was taken from Allende Prieto et al. (2001). Blending of the [O I] line with the weak rotational line of $\mathrm{CN}(10,5)$ band from the red system at $6300.265 \AA$ was taken into account.

To determine the ${ }^{12} \mathrm{C} /{ }^{13} \mathrm{C}$ isotopic ratios we considered two spectral regions: 4360-4370 ̊, containing lines of ${ }^{12} \mathrm{CH}$ and ${ }^{13} \mathrm{CH}$ molecules, and 7994-8020 $\AA$ containing ${ }^{12} \mathrm{CN}$ and ${ }^{13} \mathrm{CN}$ molecule lines. Unfortunately, our spectra in the $\sim 8000 \AA$ region are strongly contaminated by telluric $\mathrm{H}_{2} \mathrm{O}$ lines. In particular, the most prominent ${ }^{13} \mathrm{CN}$ feature at $\sim 8004 \AA$ in the spectrum of HD 10613 is blended by a strong telluric $\mathrm{H}_{2} \mathrm{O} 8007.5 \AA$ line. Although we eliminated telluric lines by dividing our spectra by the spectrum of a hot high-rotating star, any uncertainties in telluric line treatment strongly affect the derived value of ${ }^{12} \mathrm{C} /{ }^{13} \mathrm{C}$ ratio. This is why the determination of the carbon isotopic ratio is mainly based on ${ }^{13} \mathrm{CH}$ lines in the $4360 \AA$ spectral region. We consider three ${ }^{13} \mathrm{CH}$ features at 4363.8, 4366.1, and $4367.2 \AA$. The derived carbon isotopic ratios are ${ }^{12} \mathrm{C} /{ }^{13} \mathrm{C}=16_{-3}^{+8}$ for the HD 10613 and ${ }^{12} \mathrm{C} /{ }^{13} \mathrm{C}=15_{-3}^{+5}$ for $\mathrm{BD}+04^{\circ} 2466$.

Lithium abundance was derived from the Li I $6708 \AA$ resonance doublet. In our calculations we included in the line list the lines of $\mathrm{Ce}, \mathrm{Nd}$, and $\mathrm{Sm}$ in the vicinity of the Li I line from the D.R.E.A.M. database. The $\mathrm{Ce}$ and $\mathrm{Nd}$ abundances determined in this work were used. The $\mathrm{CN}$ lines in the vicinity of the Li I doublet were included in the line list. The wavelengths and oscillator strengths for the individual hyperfine and isotope components of the lithium lines were taken from Smith et al. (1998) and Hobbs et al. (1999). The solar ${ }^{6} \mathrm{Li} /{ }^{7} \mathrm{Li}$ isotopic ratio $\left({ }^{6} \mathrm{Li} /{ }^{7} \mathrm{Li}=0.081\right)$ was adopted in synthetic spectrum calculations.

\subsection{Abundance uncertainties}

Tables 5 and 6 show that neutral elements are rather sensitive to temperature variations while single ionized elements are sensitive to the variations in $\log g$. In the case of the elements whose 
Table 4. Abundance in the $\log \varepsilon(\mathrm{H})=12.0$ scale and in the notation $[\mathrm{X} / \mathrm{Fe}]$.

\begin{tabular}{|c|c|c|c|c|c|c|c|c|}
\hline \multirow[b]{2}{*}{ Species } & \multicolumn{4}{|c|}{ HD 10613} & \multicolumn{4}{|c|}{$\mathrm{BD}+04^{\circ} 2466$} \\
\hline & $\mathrm{n}$ & $\log \varepsilon$ & {$[\mathrm{X} / \mathrm{H}]$} & {$[\mathrm{X} / \mathrm{Fe}]$} & $\mathrm{n}$ & $\log \varepsilon$ & {$[\mathrm{X} / \mathrm{H}]$} & {$[\mathrm{X} / \mathrm{Fe}]$} \\
\hline Fe I & 71 & $6.70 \pm 0.11$ & -0.82 & - & 42 & $5.60 \pm 0.08$ & -1.92 & - \\
\hline Fe II & 10 & $6.67 \pm 0.03$ & -0.85 & - & 8 & $5.60 \pm 0.08$ & -1.92 & - \\
\hline$\overline{C I}$ & 2 & $8.25 \pm 0.06$ & -0.27 & +0.55 & 2 & $7.77 \pm 0.15$ & -0.75 & +1.17 \\
\hline $\mathrm{NI}_{\mathrm{I}}$ & 1 & $7.43 \pm 0.22$ & -0.49 & +0.33 & 1 & $7.10 \pm 0.25$ & -0.82 & +1.10 \\
\hline OI & 1 & $8.53 \pm 0.04$ & -0.30 & +0.52 & 1 & $7.21 \pm 0.07$ & -1.62 & +0.30 \\
\hline $\mathrm{NaI}$ & 2 & 5.35 & -0.98 & -0.16 & 2 & 4.53 & -1.80 & +0.02 \\
\hline $\operatorname{Mg} I$ & 2 & 7.03 & -0.55 & +0.27 & - & - & - & - \\
\hline Si I & 6 & $6.93 \pm 0.11$ & -0.62 & +0.20 & 2 & 6.24 & -1.31 & +0.61 \\
\hline $\mathrm{CaI}$ & 7 & $5.75 \pm 0.12$ & -0.61 & +0.21 & 11 & $4.97 \pm 0.20$ & -1.39 & +0.53 \\
\hline Sc II & 6 & $2.45 \pm 0.26$ & -0.72 & +0.10 & 5 & $1.25 \pm 0.12$ & -1.92 & 0.00 \\
\hline Ti I & 8 & $4.42 \pm 0.12$ & -0.60 & +0.22 & 2 & 3.27 & -1.75 & +0.17 \\
\hline $\mathrm{CrI}$ & 6 & $4.97 \pm 0.12$ & -0.70 & +0.12 & 4 & $3.63 \pm 0.06$ & -2.04 & -0.12 \\
\hline Mn I & 2 & 4.29 & -1.10 & -0.28 & 1 & 3.09 & -2.30 & -0.38 \\
\hline Ni I & 10 & $5.43 \pm 0.08$ & -0.82 & 0.00 & 3 & $4.13 \pm 0.18$ & -2.12 & -0.20 \\
\hline $\mathrm{Cu} \mathrm{I}$ & 1 & 3.51 & -0.70 & +0.12 & - & - & - & - \\
\hline $\mathrm{ZnI}$ & 1 & 3.72 & -0.88 & -0.06 & 2 & 2.92 & -1.68 & +0.24 \\
\hline Y II & 7 & $2.20 \pm 0.20$ & -0.04 & +0.78 & 6 & $0.79 \pm 0.13$ & -1.45 & +0.47 \\
\hline ZrI & 3 & $2.71 \pm 0.05$ & +0.11 & +0.93 & - & - & - & - \\
\hline $\mathrm{Zr}$ II & 2 & $2.61 \pm 0.43$ & 0.00 & +0.82 & 4 & $1.47 \pm 0.19$ & -1.13 & +0.79 \\
\hline Ba II & 1 & 2.75 & +0.62 & +1.44 & 1 & 1.91 & -0.22 & +1.70 \\
\hline La II & 6 & $1.67 \pm 0.23$ & +0.50 & +1.32 & 4 & $0.45 \pm 0.05$ & -0.72 & +1.20 \\
\hline Ce II & 6 & $1.91 \pm 0.20$ & +0.33 & +1.15 & 5 & $0.73 \pm 0.15$ & -0.85 & +1.07 \\
\hline Nd II & 16 & $2.23 \pm 0.21$ & +0.73 & +1.55 & 12 & $0.93 \pm 0.12$ & -0.57 & +1.35 \\
\hline Eu II & 1 & 0.41 & -0.10 & +0.72 & - & - & - & - \\
\hline $\mathrm{Pb} \mathrm{I}$ & 1 & 2.48 & +0.48 & +1.30 & 1 & 2.00 & 0.00 & +1.92 \\
\hline \multicolumn{4}{|c|}{$\mathrm{C} / \mathrm{O}=0.52 \pm 0.09$} & \multicolumn{5}{|c|}{$\mathrm{C} / \mathrm{O}=3.63 \pm 0.23$} \\
\hline
\end{tabular}

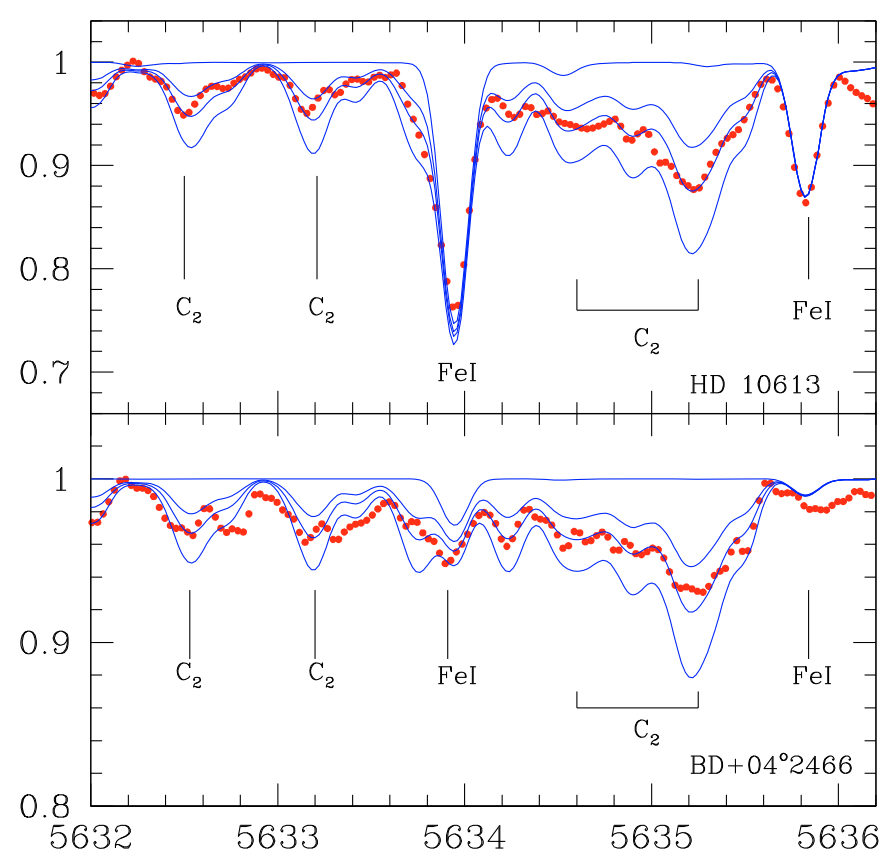

Fig. 3. Observed (dotted red line) and synthetic (solid blue line) spectra in the region around the $\mathrm{C}_{2}$ molecule lines at $5635 \AA$ for the stars HD 10613 and $\mathrm{BD}+04^{\circ} 2466$. In the synthetic spectra of HD 10613 we show the synthesis for carbon abundances of $\log \varepsilon(\mathrm{C})=$ none, 8.25, 8.35 and 8.45 and for $\mathrm{BD}+04^{\circ} 2466$, for carbon abundances of $\log \varepsilon(\mathrm{C})=$ none, 7.71, 7.81 and 7.91 .

abundance is based on stronger lines, such as barium, the error introduced by the microturbulence is significant. For the elements analyzed via spectrum synthesis the same technique was used, varying $T_{\text {eff }}, \log g$ and $\xi$, then independently computing the abundance changes introduced by the variation of the above atmospheric parameters. They are also included in Tables 5 and 6.

The abundance uncertainties due to the errors in the equivalent width measurements were computed from an expression provided by Cayrel (1988). The errors in the equivalent widths are set, essentially, by the signal-to-noise ratio and the spectral resolution. In our case, having $R \approx 48000$ and a typical $S / N$ ratio of 150 , the expected uncertainties in the equivalent widths are about 2-3 $\mathrm{m} \AA$. These error estimates have been applied to the measured $W_{\lambda}^{\prime}$ s and the corresponding changes in the element abundances are listed in Col. 5 of Tables 5 and 6.

We also estimated the influence of model errors, such as uncertainties in the effective temperatures and surface gravities, on the derived CNO abundances. Moreover, as we have mentioned above, abundances of the CNO elements are interdependent, so uncertainties in the oxygen abundance determination affect the carbon abundance and vice versa. Uncertainties in the carbon abundance result in variation of nitrogen abundances, since the $\mathrm{CN}$ molecular lines were used for the $\mathrm{N}$ abundance determination. The variations of the abundance due to changes in effective temperature $( \pm 120 \mathrm{~K})$, surface gravity $( \pm 0.2$ dex $)$ and $\mathrm{C}, \mathrm{N}$, and $\mathrm{O}$ abundances are summarized in Tables 8 and 9 for HD 10613 and $\mathrm{BD}+04^{\circ} 2466$. In the last column we present the resulting abundance uncertainties, $\sigma_{\text {tot }}$, calculated as the square root of the squares of the various sources of uncertainty. Derived CNO abundances are weakly sensitive to the variations of the microturbulent velocity since weak lines were used for their determination. The other source of uncertainty in the molecular line analysis is the uncertainty in the molecular constants, mainly the dissociation energy of the CN molecule which is not yet well known (Lambert 1994). Our tests showed that variations of the $\mathrm{CN}$ molecule dissociation energy of $\Delta D_{0}(\mathrm{CN})=+0.10 \mathrm{eV}$ resulted in variations of nitrogen abundance $\Delta \log \varepsilon(\mathrm{N})=-0.10$ dex. Calculations of the 
Table 5. Abundance uncertainties of HD 10613. The second column gives the variation of the abundance caused by the variation in $T_{\mathrm{eff}}$. The other columns refer, respectively, to the variations due to $\log g$, $\xi$ and $W_{\lambda}$. The sixth column gives the compounded rms uncertainty of the second to fifth columns. The last column gives the observed abundance dispersion for those elements which abundances were derived using more than three lines.

\begin{tabular}{lcccccc}
\hline \hline Species & $\Delta T_{\text {eff }}$ & $\Delta \log g$ & $\Delta \xi$ & $\Delta W_{\lambda}$ & $\left(\sum \sigma^{2}\right)^{1 / 2}$ & $\sigma_{\text {obs }}$ \\
& $+120 \mathrm{~K}$ & +0.1 & +0.2 & $3 \mathrm{~m} \AA$ & & \\
\hline Fe I & +0.12 & +0.05 & -0.05 & +0.02 & 0.18 & 0.11 \\
Fe II & -0.05 & +0.12 & 0.00 & +0.05 & 0.14 & 0.03 \\
Na I & +0.16 & -0.01 & -0.02 & +0.02 & 0.16 & - \\
Mg I & +0.13 & +0.01 & -0.02 & +0.04 & 0.14 & - \\
Si I & +0.04 & +0.01 & -0.01 & -0.03 & 0.11 & 0.11 \\
Ca I & +0.07 & -0.01 & -0.08 & +0.04 & 0.17 & 0.12 \\
Sc II & -0.05 & +0.03 & +0.05 & +0.04 & 0.07 & 0.26 \\
Ti I & +0.11 & 0.00 & -0.06 & +0.04 & 0.21 & 0.12 \\
Cr I & +0.11 & 0.00 & -0.10 & +0.03 & 0.22 & 0.12 \\
Mn I & +0.15 & 0.00 & -0.05 & +0.03 & 0.21 & - \\
Ni I & +0.11 & +0.01 & -0.05 & +0.03 & 0.12 & 0.08 \\
Cu I & +0.13 & +0.01 & +0.04 & -0.02 & 0.14 & 0.08 \\
Zn I & +0.06 & +0.03 & -0.08 & -0.02 & 0.13 & - \\
Y II & +0.04 & +0.04 & -0.09 & -0.07 & 0.12 & 0.15 \\
Zr I & +0.19 & 0.00 & -0.01 & +0.03 & 0.21 & - \\
Zr II & +0.05 & +0.07 & -0.03 & +0.05 & 0.09 & 0.43 \\
Ba II & +0.11 & +0.10 & -0.11 & -0.10 & 0.18 & - \\
La II & +0.07 & +0.11 & -0.04 & -0.04 & 0.14 & 0.23 \\
Ce II & +0.06 & -0.10 & -0.04 & +0.05 & 0.12 & 0.20 \\
Nd II & +0.00 & +0.09 & -0.10 & +0.03 & 0.15 & 0.21 \\
Eu II & +0.02 & +0.10 & +0.04 & -0.02 & 0.11 & 0.21 \\
\hline & & & & & &
\end{tabular}

carbon isotopic ratios do not depend on the uncertainties in the $\mathrm{C}$ and $\mathrm{N}$ abundances and molecular parameters. The errors in the ${ }^{12} \mathrm{C} /{ }^{13} \mathrm{C}$ determinations are mainly due to uncertainties in the observed spectra, such as possible contamination by unidentified atomic or molecular lines, or uncertainties in the continuum placement.

\section{Discussion}

\subsection{Spectral type and distance of $H D 10613$ and $B D+04^{\circ} 2466$}

\subsubsection{HD 10613}

Previous spectroscopic observations of HD 10613 were performed by MacConnel et al. (1972) and Lu (1991) with photographic plates. Section 3.2 showed that the computed temperature is similar to the one found by these authors. MacConnel et al. (1972) classified HD 10613 as a K0 star and Lu (1991) as a K1 III star.

The relation between temperature, gravity, mass, $V$ magnitude, interstellar absorption $\left(A_{V}\right)$, and the bolometric correction $(B C)$ is given by:

$$
\begin{aligned}
\log r(\mathrm{kpc})= & \frac{1}{2}\left(\log \frac{M_{\star}}{M_{\odot}}+0.4\left(V-A_{V}+B C\right)\right. \\
& \left.+4 \log T_{\text {eff }}-\log g-16.5\right) .
\end{aligned}
$$

Inserting the values of $T_{\text {eff }}=5100 \mathrm{~K}$ and $\log g=2.8$ and assuming for the mass $M_{*} \simeq M_{\odot}$, this equation becomes

$5 \log r(\mathrm{kpc})=\left(V-A_{V}+B C\right)-11.17$.

Considering $V=9.6$ (MacConnel et al. 1972) and assuming $A_{V}=0.0$ for a halo star and $B C=-0.25$, which is the bolometric
Table 6. Abundance uncertainties of $\mathrm{BD}+04^{\circ} 2466$. The second column gives the variation of the abundance caused by the variation in $T_{\mathrm{eff}}$. The other columns refer to the variations due to $\log g, \xi$ and $W_{\lambda}$. The sixth column gives the compounded rms uncertainty of the second to fifth columns. The last column gives the observed abundance dispersion for those elements whose abundances were derived using more than three lines.

\begin{tabular}{lcccccc}
\hline \hline Species & $\Delta T_{\text {eff }}$ & $\Delta \log g$ & $\Delta \xi$ & $\Delta W_{\lambda}$ & $\left(\sum \sigma^{2}\right)^{1 / 2}$ & $\sigma_{\text {obs }}$ \\
& $+120 \mathrm{~K}$ & +0.1 & +0.2 & $3 \mathrm{~m} \AA$ & & \\
\hline Fe I & +0.10 & -0.01 & +0.08 & +0.07 & 0.15 & 0.08 \\
Fe II & +0.01 & +0.09 & -0.05 & +0.16 & 0.19 & 0.08 \\
Na I & +0.05 & 0.00 & -0.01 & +0.05 & 0.07 & - \\
Si I & +0.04 & 0.00 & -0.01 & +0.09 & 0.10 & - \\
Ca I & +0.11 & -0.01 & -0.07 & +0.04 & 0.14 & 0.20 \\
Sc II & -0.02 & +0.06 & -0.03 & +0.04 & 0.08 & 0.12 \\
Ti I & +0.09 & -0.01 & -0.10 & +0.04 & 0.14 & - \\
Cr I & +0.19 & -0.01 & -0.04 & +0.04 & 0.20 & 0.06 \\
Mn I & +0.20 & +0.01 & -0.05 & +0.03 & 0.21 & - \\
Ni I & +0.11 & +0.01 & -0.01 & +0.05 & 0.12 & 0.18 \\
Zn I & -0.11 & +0.02 & -0.04 & +0.04 & 0.13 & - \\
Y II & 0.00 & +0.05 & -0.10 & +0.04 & 0.06 & - \\
Zr II & +0.01 & +0.07 & -0.05 & +0.05 & 0.10 & 0.19 \\
Ba II & +0.04 & +0.10 & -0.20 & +0.05 & 0.23 & - \\
La II & +0.02 & +0.11 & -0.02 & +0.04 & 0.12 & 0.05 \\
Ce II & +0.01 & -0.10 & -0.02 & +0.05 & 0.11 & 0.15 \\
Nd II & +0.02 & +0.09 & -0.04 & +0.07 & 0.12 & 0.12 \\
\hline
\end{tabular}

Table 7. Influence of errors on the $\mathrm{CNO}$ abundances derived for HD 10613.

\begin{tabular}{lrrrccc}
\hline \hline Species & $\begin{array}{c}\Delta T_{\text {eff }} \\
+120 \mathrm{~K}\end{array}$ & $\begin{array}{c}\Delta \log g \\
+0.1\end{array}$ & $\begin{array}{r}\Delta \xi_{\mathrm{m}} \\
+0.2\end{array}$ & $\begin{array}{c}\Delta \log (\mathrm{C}) \\
+0.1\end{array}$ & $\begin{array}{c}\Delta \log (\mathrm{O}) \\
+0.1\end{array}$ & $\sigma_{\text {tot }}$ \\
\hline $\mathrm{C}$ & +0.04 & +0.01 & 0.00 & - & +0.03 & 0.05 \\
$\mathrm{~N}$ & +0.17 & 0.00 & 0.00 & -0.10 & +0.05 & 0.20 \\
$\mathrm{O}$ & 0.00 & +0.03 & +0.01 & 0.00 & - & 0.03 \\
\hline
\end{tabular}

Table 8. Influence of errors on the $\mathrm{CNO}$ abundances derived for $\mathrm{BD}+04^{\circ} 2466$.

\begin{tabular}{lccrccc}
\hline \hline Species & $\begin{array}{c}\Delta T_{\text {eff }} \\
+100 \mathrm{~K}\end{array}$ & $\begin{array}{c}\Delta \log g \\
+0.2\end{array}$ & $\begin{array}{r}\Delta \xi_{\mathrm{m}} \\
+0.2\end{array}$ & $\begin{array}{c}\Delta \log (\mathrm{C}) \\
+0.15\end{array}$ & $\begin{array}{c}\Delta \log (\mathrm{O}) \\
+0.15\end{array}$ & $\sigma_{\text {tot }}$ \\
\hline $\mathrm{C}$ & +0.15 & -0.02 & -0.01 & - & 0.00 & 0.15 \\
$\mathrm{~N}$ & +0.21 & -0.04 & 0.00 & -0.12 & +0.01 & 0.24 \\
$\mathrm{O}$ & +0.05 & +0.05 & -0.01 & 0.00 & - & 0.07 \\
\hline
\end{tabular}

correction given by Alonso et al. (1999) for giant stars with metallicity of $[\mathrm{Fe} / \mathrm{H}]=-1.0$, Eq. (2) then gives $r \simeq 430 \mathrm{pc}$. The bolometric magnitude which results from the distance derived above is $M_{\mathrm{bol}} *=+1.17$ and the luminosity is $L_{*} \simeq 30 L_{\odot}$ assuming $M_{\text {bol } \odot}=+4.74$ for the Sun (Bessel 1998). The obtained luminosity of HD 10613 is too low to be an asymptotic giant branch star that could have commenced shell helium burning (via thermal pulses) and became self-enriched in the neutroncapture elements.

Our spectroscopic gravity determined from ionization equilibrium agrees well with that derived using the distance given by Mennessier et al. (1997) of 524 pc. HD 10613 displays characteristics of a halo object, as was already claimed by Goméz et al. (1997). It is a low metallicity object and has high radial velocity and high galactic latitude (see Table 2). 


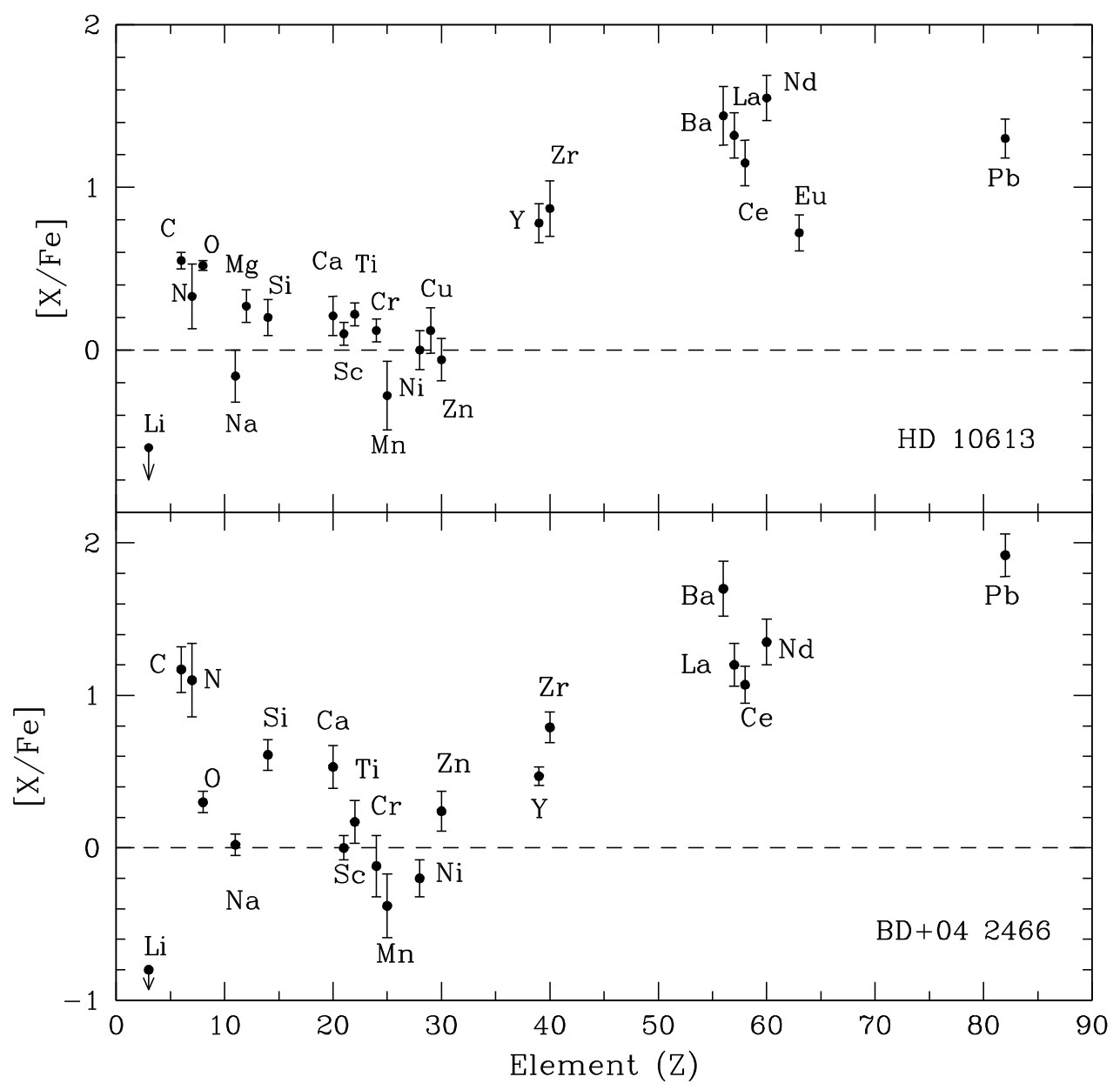

Fig. 4. Abundance pattern of $\mathrm{HD} 10613$ a) and of $\mathrm{BD}+04^{\circ} 2466 \mathbf{b}$ ). Error bars represent the uncertainty estimates described in the text.

\subsection{2. $\mathrm{BD}+04^{\circ} 2466$}

$\mathrm{BD}+04^{\circ} 2466$ was noticed as a metal-poor star by Bond (1980) after a objective-prism survey and was later classified as a G5 star by Ruelas-Mayorga (1997). Section 3.2 showed that the derived temperature is similar to the spectral classification given above.

Inserting the values of $T_{\mathrm{eff}}=5100 \mathrm{~K}$ and $\log g=1.8$, and assuming for the mass $M_{*} \simeq M_{\odot}$, Eq. (1) becomes

$5 \log r(\mathrm{kpc})=\left(V-A_{V}+B C\right)-8.67$.

Considering $V=10.5$ (Luck \& Bond 1991) and assuming $A_{V}=0.0$ for a halo star and $B C=-0.27$, which is the bolometric correction given by Alonso et al. (1999) for giant stars with metallicity of $[\mathrm{Fe} / \mathrm{H}]=-2.0$, Eq. (2) then gives $r \simeq 2.0 \mathrm{kpc}$. The bolometric magnitude which results from the distance derived above is $M_{\mathrm{bol}} *=-1.32$. The obtained luminosity is $L_{*} \simeq 270 L_{\odot}$ assuming $M_{\text {bol } \odot}=+4.74$ for the Sun (Bessel 1998). Although $\mathrm{BD}+04^{\circ} 2466$ presents a higher luminosity than HD 10613 its luminosity is not high enough to be considered as an asymptotic giant branch star that could have commenced shell helium burning.

Table 4 shows that $\mathrm{BD}+04^{\circ} 2466$ is a $\mathrm{CH}$ star, since its carbon-to-oxygen ratio $\mathrm{C} / \mathrm{O} \geq 1.0$. In fact, with an absolute magnitude of $M_{V}=-1.05$, this value lies in the range of other $\mathrm{CH}$ stars having, according to Table IV of Hartwick \& Cowley (1985), $M_{V}$ values between -0.5 and -2 .1. Like all the $\mathrm{CH}$ stars, $\mathrm{BD}+04^{\circ} 2466$ displays characteristics of the halo population: low metallicity, high radial velocity and high galactic latitude.

\subsection{Abundances}

Below we discuss the abundance pattern of studied stars comparing it with previous studies done for some stars in the halo, and also with the abundance patterns of chemically peculiar stars where the heavy-element overabundances have already been reported in the literature. Figure 4 shows the abundance pattern of both stars analyzed in this work.

\subsubsection{The $\log \mathrm{C} / \mathrm{N}-\log \mathrm{O} / \mathrm{N}$ diagram}

In Fig. 5 we show the $\log \mathrm{O} / \mathrm{N}$ ratio versus the $\log \mathrm{C} / \mathrm{N}$ ratio for several classes of chemically peculiar objects for which CNO abundances have already been calculated. The solid line represents the $\mathrm{C} / \mathrm{O}=1.0$. In addition, the classical galactic carbon stars as well as the post-AGB stars were included in the diagram with the aim to show where the carbon enriched objects lie. Since barium stars are also giants but with some degree of carbon enrichment, its position in the $\log \mathrm{O} / \mathrm{N}$ versus the $\log \mathrm{C} / \mathrm{N}$ diagram should not be the same as the non-enriched GK giants.

The position of HD 10613 in Fig. 5 in the right upper corner, but at the left of the $\mathrm{C} / \mathrm{O}=1$ line, gives support for the classification of this star as a barium star. More interesting is the position of $\mathrm{BD}+04^{\circ} 2466$ in this diagram. We can see that it displays light element abudance ratios that are similar to those of $\mathrm{CH}$ stars already analyzed (Vanture 1992b) despite the scatter seen for such stars. 


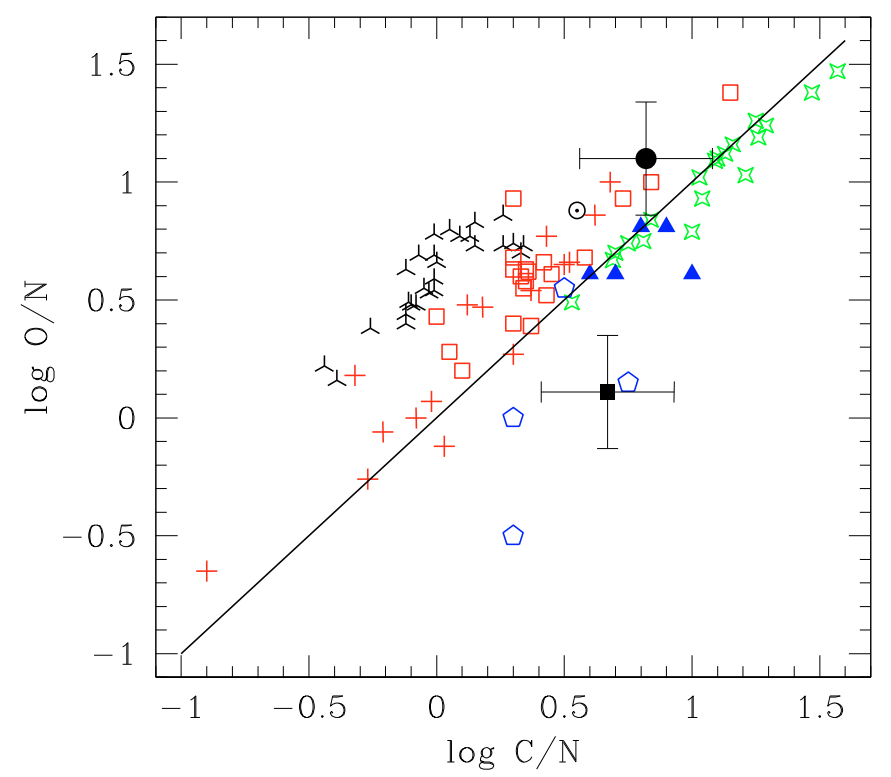

Fig. 5. Relative abundance $\mathrm{O} / \mathrm{N}$ versus $\mathrm{C} / \mathrm{N}$. Disk carbon stars (green star shaped points); GK giants (upside-down "Y"); post-AGB stars enriched in the s-process elements (blue filled triangles); barium giants (red open squares); $\mathrm{CH}$ stars (blue open polygons); subgiant $\mathrm{CH}$ stars (red plus sign). The stars analyzed in this work HD 10613 (filled black circle) and $\mathrm{BD}+04^{\circ} 2466$ (filled black square). Abundance data for barium giant and dwarf stars are from Smith (1984), Barbuy et al. (1992), Allen \& Barbuy (2006), Drake \& Pereira (2007) and Sneden et al. (1981); CH stars from Vanture (1992b); disk carbon stars from Lambert et al. (1986); GK giants from Lambert \& Ries (1981) and postAGB stars from van Winckel \& Reyniers (2000).

\subsubsection{Nitrogen and oxygen}

Abundance surveys of dwarf stars show that there is no trend for the $[\mathrm{N} / \mathrm{Fe}]$ ratio versus $[\mathrm{Fe} / \mathrm{H}]$, that is, in the metallicity range $+0.3>[\mathrm{Fe} / \mathrm{H}]>-2.0$ the $[\mathrm{N} / \mathrm{Fe}]$ is $\approx 0.0$ (Clegg et al. 1981; Tomkin \& Lambert 1984; Carbon et al. 1987). However, as a star becomes giant, due to deepening of its convective envelope, nuclear processed material is brought from the interior to the outer layers of the star changing the surface composition. As a consequence of the first dredge-up process, the abundance of ${ }^{12} \mathrm{C}$ is reduced and the abundance of nitrogen is enhanced (Lambert 1981).

In the barium giant HD 10613 the $[\mathrm{N} / \mathrm{Fe}]$ ratio is $0.33 \pm 0.25$. This ratio is similar to that found in other previous studies of barium giant, a mean $\langle[\mathrm{N} / \mathrm{Fe}]\rangle$ ratio already obtained is $0.44 \pm$ $0.18,0.58 \pm 0.16,0.47 \pm 0.12$ and $0.34 \pm 0.36$ respectively from Sneden et al. (1981), Smith (1984), Barbuy et al. (1992) and Allen \& Barbuy (2006). In addition, the value of [N/Fe] ratio for HD 10613 is also similar to the same ratio observed in $\mathrm{G}$ and $\mathrm{K}$ giants of $0.37 \pm 0.07$ (Lambert \& Ries 1981).

The $[\mathrm{N} / \mathrm{Fe}]$ ratio in $\mathrm{BD}+04^{\circ} 2466$ is very high, $1.1 \pm 0.26$. A similar ratio has been found for the $\mathrm{CH}$ stars analyzed by Vanture (1992c), $1.3 \pm 0.6$. Like HD 206983 (Drake \& Pereira 2008) this large value of the $[\mathrm{N} / \mathrm{Fe}]$ ratio in $\mathrm{BD}+04^{\circ} 2466$ is taken as an evidence of the first dredge-up as well as an efficiency of mixing since it is a metal-poor star.

The oxygen abundances in both stars follow the same trend as seen for halo stars of such metallicity. The mean $[\mathrm{O} / \mathrm{Fe}]$ for a star with a metallicity in the range between -1.0 and -2.0 is $\approx+0.5$ (Masseron et al. 2006).

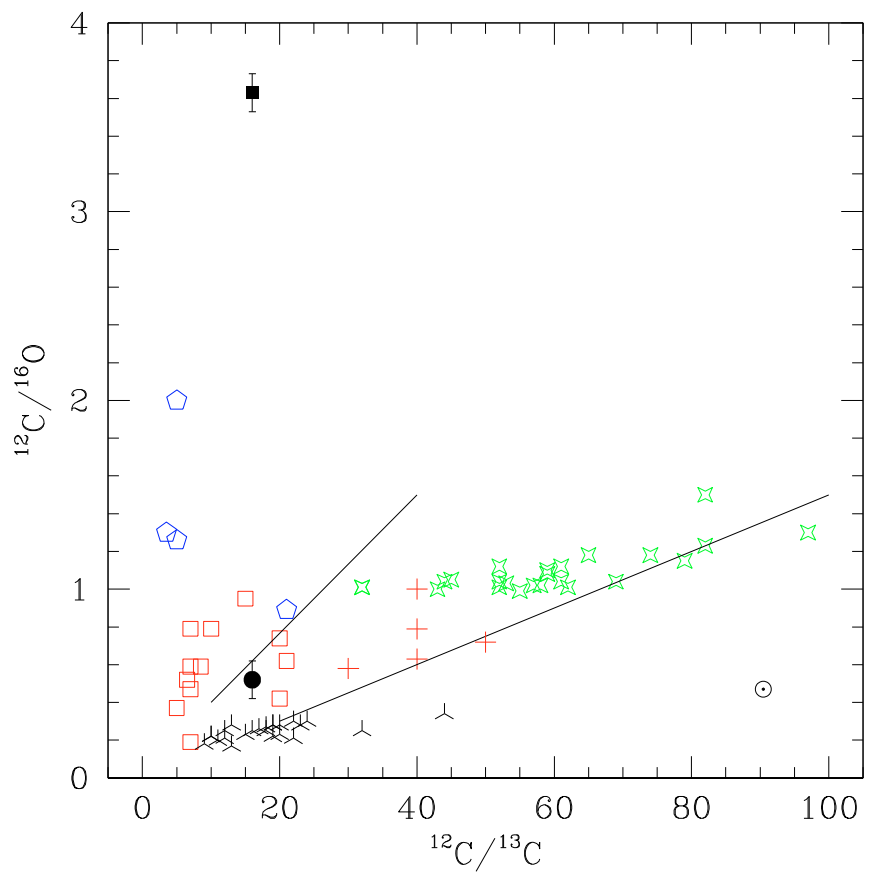

Fig. 6. ${ }^{12} \mathrm{C} /{ }^{16} \mathrm{O}$ versus ${ }^{12} \mathrm{C} /{ }^{13} \mathrm{C}$ ratios for several classes of stars and the stars analyzed in this work. Symbols have the same meaning as in Fig. 5. The solid lines represent the addition of pure ${ }^{12} \mathrm{C}$ to ${ }^{12} \mathrm{C} /{ }^{16} \mathrm{O}$ and ${ }^{12} \mathrm{C} /{ }^{13} \mathrm{C}$ ratios which are found at the bottom of the GKM giants location.

\subsection{3. ${ }^{12} \mathrm{C} /{ }^{13} \mathrm{C}$ ratio}

In Fig. 6, we show the ${ }^{12} \mathrm{C} /{ }^{16} \mathrm{O}$ versus ${ }^{12} \mathrm{C} /{ }^{13} \mathrm{C}$. In this figure we show the stars analyzed in this work together with the same classes of stars of Fig. 5, the GK giants, the disk carbon stars, the barium giants, the $\mathrm{CH}$ stars and subgiant $\mathrm{CH}$ stars. The two straight lines whose values were taken from Smith \& Lambert (1990) represent the addition of ${ }^{12} \mathrm{C}$ material on the atmosphere of a star, in a region of the diagram of Fig. 6, one starting at $\left({ }^{12} \mathrm{C} /{ }^{13} \mathrm{C},{ }^{12} \mathrm{C} /{ }^{16} \mathrm{O}\right)=(10,0.4)$ and the other at $\left({ }^{12} \mathrm{C} /{ }^{13} \mathrm{C}\right.$, $\left.{ }^{12} \mathrm{C} /{ }^{16} \mathrm{O}\right)=(20,0.3)$. According to these authors, an increase of ${ }^{12} \mathrm{C}$ by 2.5 times is necessary to change an M-type star to C-type star, as expected by the third dredge-up. Then, these two straight lines represent the limits given by the distribution of $M$ stars where an addition of ${ }^{12} \mathrm{C}$ in their atmospheres would change them from M-stars to C-stars (Smith \& Lambert 1990).

In Fig. 6 barium stars (red open squares) occupy a region between the GK-giants and $\mathrm{C}$ stars. They closely follow the sequence which GK giants follow passing through the $S$ and SC stars to become a carbon star along the TP-AGB phase. In $\mathrm{CH}$ subgiants the ${ }^{12} \mathrm{C} /{ }^{13} \mathrm{C}$ ratio has been investigated by Sneden (1983) and Drake \& Pereira (2007). These two studies show that the ${ }^{12} \mathrm{C} /{ }^{13} \mathrm{C}$ ratio lies between 20 and 40 . Compared to giants, this is taken as an evidence that the first dredge-up has not yet started in these stars. In fact, in $\delta$ Eri, a subgiant analyzed by Lambert \& Ries (1981), the ${ }^{12} \mathrm{C} /{ }^{13} \mathrm{C}$ ratio is greater than 50. In normal giants the ${ }^{12} \mathrm{C} /{ }^{13} \mathrm{C}$ ratio is $17.5 \pm 0.2$ (Lambert \& Ries 1981), which is close to the predictions, 20-30 (Iben \& Renzini 1983). The position of $\mathrm{CH}$ subgiants in Fig. 6, between the normal GK giants and the carbon stars, also suggests that the $\mathrm{CH}$ subgiant stars may have acquired substantial amounts of ${ }^{12} \mathrm{C}$ from a S- or $\mathrm{N}$-type star with ${ }^{12} \mathrm{C} /{ }^{13} \mathrm{C}$ ratio of $20-100$ (Smith et al. 1993). According to these authors the ${ }^{12} \mathrm{C} /{ }^{13} \mathrm{C}$ ratio observed in the $\mathrm{CH}$ subgiants would be consistent with the hypothesis that the $\mathrm{CH}$ subgiants evolve into barium giants. 
The ${ }^{12} \mathrm{C} /{ }^{13} \mathrm{C}$ ratio in barium giants has been investigated by Smith (1984, 1992), Barbuy et al. (1992), Sneden et al. (1981) and Drake \& Pereira (2008) and in CH stars by Vanture (1992b). For barium giants the ${ }^{12} \mathrm{C} /{ }^{13} \mathrm{C}$ ratio lies between 8 and 20 . For the CH stars, Vanture (1992c) showed that they could be divided into two groups, according to the ${ }^{12} \mathrm{C} /{ }^{13} \mathrm{C}$ ratio, some having ${ }^{12} \mathrm{C} /{ }^{13} \mathrm{C} \approx 3.0$ and others with ${ }^{12} \mathrm{C} /{ }^{13} \mathrm{C} \geq 25.0$. Inspecting Fig. 6, it seems that, like the $\mathrm{CH}$ stars, the barium giants could also be divided into two groups according to their ${ }^{12} \mathrm{C} /{ }^{13} \mathrm{C}$ ratios, a few having ${ }^{12} \mathrm{C} /{ }^{13} \mathrm{C}$ between 5 and 10 and a some having ${ }^{12} \mathrm{C} /{ }^{13} \mathrm{C} \approx$ $20-25$. Those that have lower carbon isotopic ratios lie leftward of the upper straight line that divides an M stars from C stars. Probably, but this remains to be verified when the masses of barium stars become available, that division in the ${ }^{12} \mathrm{C} /{ }^{13} \mathrm{C}$ ratios maybe related to mass.

In barium giants, the low carbon isotopic ratio is currently explained by two mixing episodes, the first dredge-up and the mixing due to inversion of the mean molecular weight (Barbuy et al. 1992). This non-canonical mixing or thermohaline convection has been suggested as a possible source of mixing while the star is still on the main sequence or at the subgiant phase, before the occurrence of the first dredge-up, since the C-rich material, which is deposited at the surface of an accreting star during the mass transfer, has a mean molecular weight greater than that of the unevolved star (Stancliffe et al. 2007; Denissenkov \& Pinsonneault 2008).

The two stars analyzed in this work, with ${ }^{12} \mathrm{C} /{ }^{13} \mathrm{C}=16$ (HD 10613) and $15\left(\mathrm{BD}+04^{\circ} 2466\right)$, add only two data points in this diagram so that a determination of the ${ }^{12} \mathrm{C} /{ }^{13} \mathrm{C}$ ratio in barium stars would be very important to understand the physics of the dredge-up and mixing phenomena in these binary systems.

\subsubsection{Li}

For the two stars analyzed in this work, we determined upper limits of lithium abundance. The Li abundance of HD 10613 is $\log \varepsilon(\mathrm{Li}) \leq 0.5$ and that of $\mathrm{BD}+04^{\circ} 2466$ is $\log \varepsilon(\mathrm{Li}) \leq 0.3$. Creation of the D.R.E.A.M. database permitted to identify various rare-earth-element lines contributing to the absorption feature at $6708 \AA$ in the spectra of the s-process element enriched stars. Thus, the line at $6708.099 \AA$ in the spectra of the low-mass post-AGB stars enriched in s-process elements considered previously as a "shifted Li line" (Začs et al. 1995; Reddy et al. 1997, 1999, 2002) was identified as the Ce II transition at $6708.099 \AA$ (Reyniers et al. 2002). Both stars, HD 10613 and BD+04 ${ }^{\circ} 2466$, clearly show this line in the spectra. However, the feature at $6707.740 \AA$ found in the spectra of barium stars by Lambert et al. (1993) is still not identified. This line is clearly observed in the spectrum of the more metal-deficient star BD+04 2466 . We were unable to fit this feature with the line of an ion of a heavy element, supposed by Lambert et al. (1993) to be a Ce II line, using the line parameter values (the oscillator strength and the excitation potential) suggested by Lambert et al. (1993) and the cerium abundance of $\mathrm{BD}+04^{\circ} 2466$ obtained in this work. We included the lines of the rare-earth elements identified around the Li I line by Shavrina et al. (2003) in the spectrum of the roAp star HD 101065 (Przybylski's star) highly enriched in the rareearth elements. However, the profile of the absorption feature at $6708 \AA$ cannot be fitted convincingly. Thus, creation of a complete line list in the vicinity of the Li I $6708 \AA$ line which could permit a reliable determination of the lithium abundance in the s-process enriched stars remains an open problem.

\subsubsection{Other elements: $\mathrm{Na}$ to $\mathrm{Zn}$ and $\mathrm{Eu}$}

The abundances of light odd elements, $\mathrm{Na}$ and $\mathrm{Al}$, are usually referenced to $\mathrm{Mg}$ rather than $\mathrm{Fe}$, because the elements of a lighter group are thought to be synthesized by carbon burning in massive stars while Fe is mainly produced by explosive silicon burning. For a sample of dwarfs and giants with similar metallicity analyzed by Gratton \& Sneden (1987), the typical ratio at $[\mathrm{Fe} / \mathrm{H}] \approx-1.0$ is $[\mathrm{Na} / \mathrm{Mg}]=-0.5$. For HD 10613 we obtained $[\mathrm{Na} / \mathrm{Mg}]=-0.43$. In $\mathrm{BD}+04^{\circ} 2466$ magnesium was not determined. Sodium has a typical abundance for a $[\mathrm{Fe} / \mathrm{H}] \approx-2.0$ star (Fulbright 2002).

The $[\alpha / \mathrm{Fe}]$ ratios seen in HD 10613 and in $\mathrm{BD}+04^{\circ} 2466$ are typical of halo stars of the same metallicity (Carretta et al. 2002; Ryan et al. 1991). The mean of [ $\langle\mathrm{Mg}, \mathrm{Si}, \mathrm{Ca}, \mathrm{Ti}\rangle / \mathrm{Fe}]$ for HD 10613 is $0.23 \pm 0.05$ and for $\mathrm{BD}+04^{\circ} 2466$ (without $\mathrm{Mg}$ ) is $0.44 \pm 0.33$.

Nickel, which is expected to follow iron, does indeed with $[\mathrm{Ni} / \mathrm{Fe}]=0.00$ and -0.20 respectively for HD 10613 and $\mathrm{BD}+04^{\circ} 2466$. The $[\mathrm{Ni} / \mathrm{Fe}]$ ratio stays close to 0.0 in a metallicty range from -2.0 to 0.0 (Jonsell et al. 2005). In the metallicity range $-3.0 \leq[\mathrm{Fe} / \mathrm{H}] \leq 0.0$ the $[\mathrm{Sc} / \mathrm{Fe}]$ ratio does not exhibt any trend (Carretta et al. 2002) and the stars analyzed in this work confirm this conclusion. The abundance of chromium also shows the absence of any trend in metal-poor stars. For BD $+04^{\circ} 2466$, the $[\mathrm{Cr} / \mathrm{Fe}]$ ratio has a typical value for a metal-poor star of around $[\mathrm{Fe} / \mathrm{H}] \approx-2.0$. The $\mathrm{Mn}$ deficiency is another indication that $\mathrm{HD} 10613$ and $\mathrm{BD}+04^{\circ} 2466$ are halo stars (Gratton 1989; Carretta 2002).

Copper and zinc have been analyzed by Sneden et al. (1991) for disk and halo stars. In HD 10613 copper is deficient and follows the trend observed by these authors for a $[\mathrm{Fe} / \mathrm{H}]=$ -1.0 metallicity star. The zinc abundance in HD 10613 also follows the trend seen in the metallicity range between -2.5 and -0.5 while in $\mathrm{BD}+04^{\circ} 2466$ zinc is slightly above the mean value given by Sneden et al. (1991).

The abundance of Eu seems to be in good agreement for a metal-poor star with the metallicity of about $[\mathrm{Fe} / \mathrm{H}] \sim-1.0$, as is the case of HD 10613. For a sample of halo stars (Gratton \& Sneden 1994) the $[\mathrm{Eu} / \mathrm{Fe}]$ ratio for a metallicity of $[\mathrm{Fe} / \mathrm{H}]=-1.0$ is +0.5 . The production of Eu is dominated by the r-process and is not expected to be enhanced in these s-process enriched stars.

\subsubsection{Carbon and s-process elements}

Figure 7 shows the $[\mathrm{s} / \mathrm{Fe}]$ ratios for HD 10613 and BD+04 2466 where "s" represents the mean of the elements created by slow neutron capture reactions (s-process): $\mathrm{Y}, \mathrm{Zr}, \mathrm{Ba}, \mathrm{La}, \mathrm{Ce}$, and $\mathrm{Nd}$. This figure shows the $[\mathrm{s} / \mathrm{Fe}]$ ratios for the barium stars (giants and dwarfs), the yellow symbiotic stars, the $\mathrm{CH}$ stars and the CEMP stars. The definition of "s" varies from author to author and in some cases depends on the quality and/or on the wavelength range of the available spectra. There are six CEMP stars that are binaries: CS 22942-019, CS 22948-027, CS 29497-030, CS 29497-034, CS 22964-161, and HE 00242523 , the data of which concerning their carbon and heavyelement $(Z>56)$ overabundances and binarity were taken from the recent results of Preston \& Sneden (2001), Sivarani et al. (2004), Barbuy et al. (2005), Lucatello et al. (2003), Thompson et al. (2008), Aoki et al. (2002), and Hill et al. (2000). As we can see from this figure, both HD 10613 and BD+04 2466 are enhanced in the s-process elements when compared to the same ratio for metal-poor field stars. In fact, the [element/Fe] ratio for each of these elements in HD 10613 and in $\mathrm{BD}+04^{\circ} 2466$ 


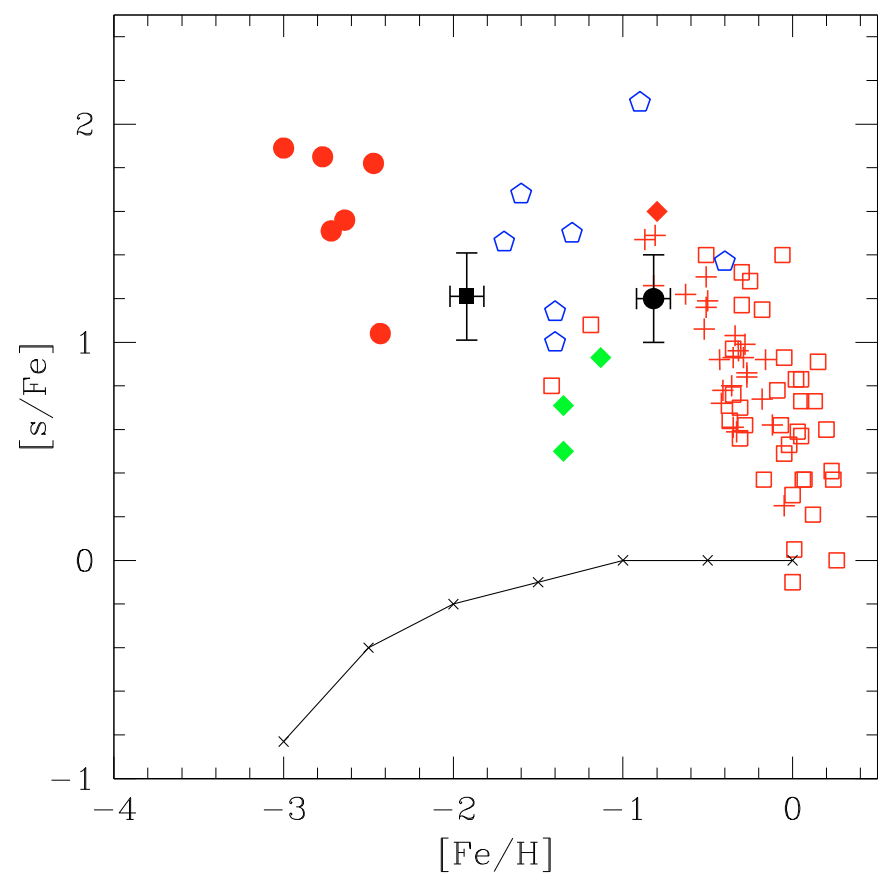

Fig. 7. Diagram of $[\mathrm{s} / \mathrm{Fe}]$ versus $[\mathrm{Fe} / \mathrm{H}]$ for several classes of chemically peculiar binary stars. Barium giants (red open squares); $\mathrm{CH}$ stars (blue open polygons); subgiant $\mathrm{CH}$ stars (red plus sign); yellow-symbiotics (green filled diamonds); $\mathrm{BD}+75^{\circ} 348$ (red filled diamond); HD 10613 (black filled circle) and $\mathrm{BD}+04^{\circ} 2466$ (black filled square). We also show some CEMP stars which are members of binary systems (red filled circles). The solid line is the mean $\langle[\mathrm{s} / \mathrm{Fe}]\rangle$ for field stars (Gratton \& Sneden 1994; Ryan et al. 1996; François et al. 2003).

(Table 4) is much higher than the same [element/Fe] ratios at the metallicities of HD 10613 and BD+04 2466 when compared to a sample of metal-poor dwarfs, subgiant and giant stars analyzed by Gratton \& Sneden (1994) and Mishenina \& Kovtyukh (2001). The mean values of $[\mathrm{Y} / \mathrm{Fe}],[\mathrm{Zr} / \mathrm{Fe}],[\mathrm{Ba} / \mathrm{Fe}],[\mathrm{La} / \mathrm{Fe}]$, $[\mathrm{Ce} / \mathrm{Fe}]$ and $[\mathrm{Nd} / \mathrm{Fe}]$ at the metallicity of HD 10613 obtained from these studies are approximately, $-0.2,+0.2,0.0,0.0,+0.2$, and +0.2 . At $[\mathrm{Fe} / \mathrm{H}]=-2.0$, these $[$ element $/ \mathrm{Fe}]$ ratios do not change significantly.

Figure 7 also shows that the $[\mathrm{s} / \mathrm{Fe}]$ ratio increases as the metallicity decreases. This is a consequence of the operation of the reaction ${ }^{13} \mathrm{C}(\alpha, n){ }^{16} \mathrm{O}$ since neutron exposure is anticorrelated with metallicity (Clayton 1988; Wallerstein 1997). As it was discussed by Busso et al. (2001) the scatter seen down to $[\mathrm{Fe} / \mathrm{H}]<-1.0$ means that the occurrence of the s-process at low metallicities is very efficient and may lead to the formation of lead. As we will see in the next section, BD+04 2466 is in fact a "lead star" according to the definition of Van Eck et al. (2003).

Figure 8 shows the $[\mathrm{C} / \mathrm{Fe}]$ abundance ratio plotted as a function of the metallicity given by $[\mathrm{Fe} / \mathrm{H}]$ for the same objects as in Fig. 7. This figure shows carbon overabundance for chemically peculiar objects which are members of binary systems and the CEMP stars as well.

\subsection{7. $\mathrm{Pb}$}

We also determined the lead abundance for HD 10613 and $\mathrm{BD}+04^{\circ} 2466$ from the $\mathrm{Pb} \mathrm{I}$ line at $\lambda 4057.81 \AA$. The line data were taken from Van Eck et al. (2003) which include isotopic shifts and hyperfine splitting. Figure 9 shows the observed and synthetic spectra for the stars analyzed in this work. In

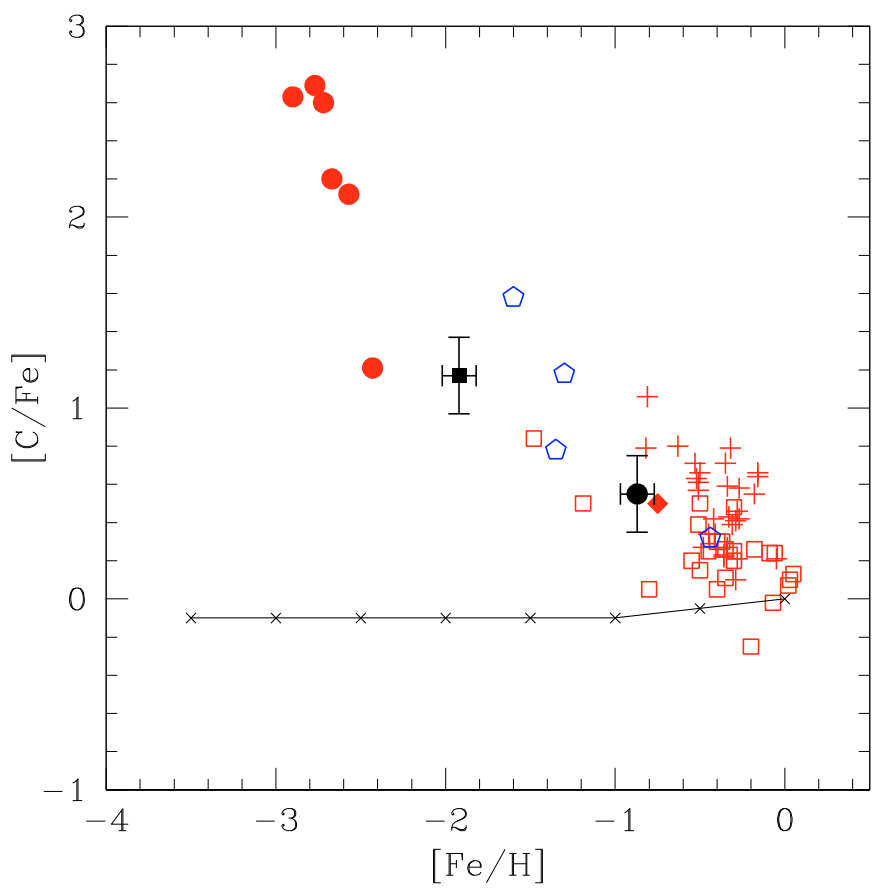

Fig. 8. Diagram of $[\mathrm{C} / \mathrm{Fe}]$ versus $[\mathrm{Fe} / \mathrm{H}]$. Symbols have the same meaning as in Fig. 7. The solid line is the mean $\langle[\mathrm{C} / \mathrm{Fe}]\rangle$ for field stars taken from Masseron et al. (2006).

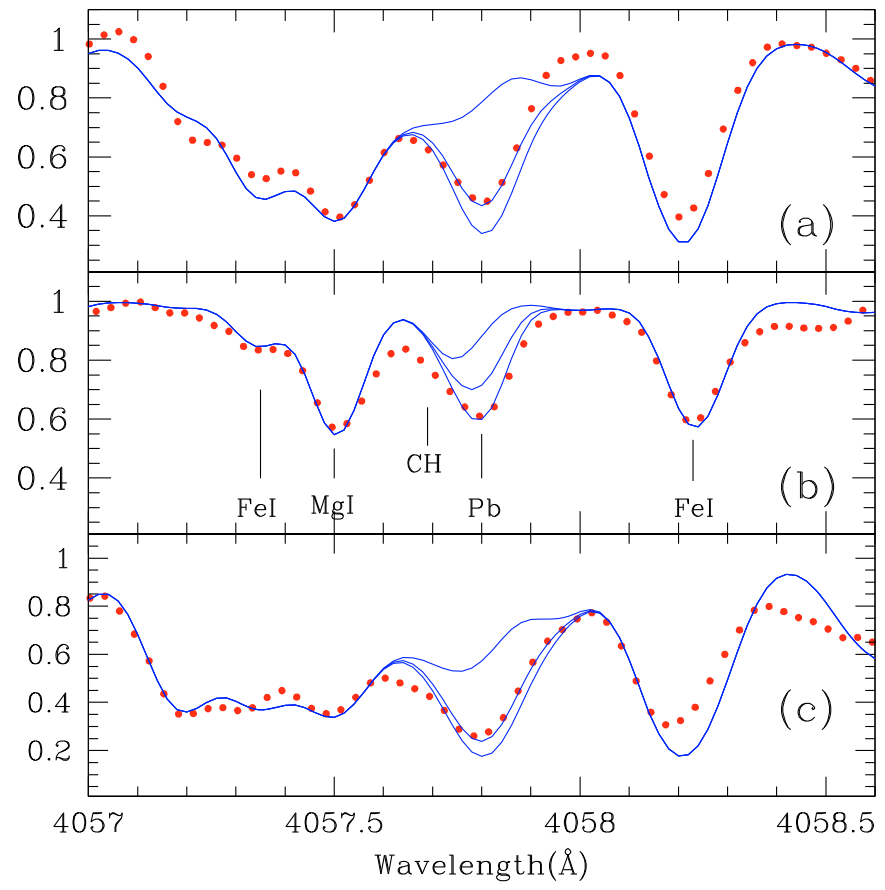

Fig. 9. Observed (dotted red line) and synthetic (solid blue line) spectra in the region around the $\mathrm{Pb}$ I line at $4057.8 \AA$ for the stars HD 10613 a), $\mathrm{BD}+04^{\circ} 2466$ b) and HD 206983 c). Synthetic spectra for HD 10613 were computed for the following lead abundances (from top to bottom): $\log \varepsilon(\mathrm{Pb})=1.03,2.48$ and 2.98. For $\mathrm{BD}+04^{\circ} 2466$, we have, respectively $\log \varepsilon(\mathrm{Pb})=-0.15,1.60$ and 2.00. For HD 206983 we have, respectively $\log \varepsilon(\mathrm{Pb})=0.39,1.79$ and 2.19 .

addition, we derived the lead abundance for the metal-poor barium star previously analyzed, HD 206983 (Junqueira \& Pereira 2001; Drake \& Pereira 2008).

In Fig. 10 we follow the Van Eck et al. (2003) study and we show the $[\mathrm{Pb} / \mathrm{Ce}]$ ratio as a function of metallicity $([\mathrm{Fe} / \mathrm{H}])$. 


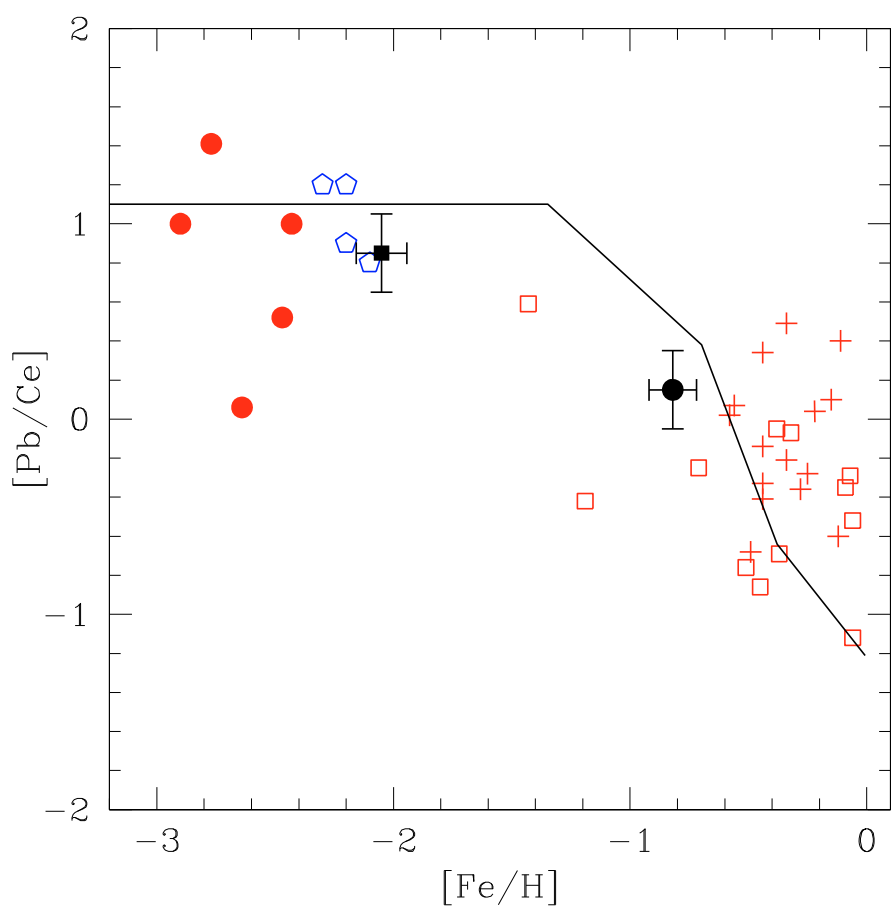

Fig. 10. The behaviour of $[\mathrm{Pb} / \mathrm{Ce}]$ ratio with metallicity for the stars analyzed in this work and HD 206983. Symbols have the same meaning as in Fig. 7, except for the CEMP star HE 0024-2523 for which there is no available $[\mathrm{Pb} / \mathrm{Ce}$ ] ratio. HD 206983 corresponds to the red open square located at $[\mathrm{Fe} / \mathrm{H}]=-1.43$.

In this figure we plot the two stars analyzed in this work, HD 206983 (the open red square at $[\mathrm{Fe} / \mathrm{H}]=-1.43$ ), the CH stars analyzed by Van Eck et al. (2003), CEMP stars which are members of binary systems (except for HE 0024-2523 for which the cerium abundance was not determined) and the barium giants and dwarfs from Allen \& Barbuy (2006). The solid line represents the prediction from the standard partial mixing (PM) model as given by Goriely \& Mowlavi (2000). The position of $\mathrm{BD}+04^{\circ} 2466$ in this diagram, close to other $\mathrm{CH}$ stars whose lead abundances were already determined, clearly indicates that it is another "lead star". Although there is a gap in this diagram for metallicities between -2.0 and -1.0 , the position of HD 206983 is interesting because it seems to follow the theoretical predictions for metallicities higher than -2.0 .

\section{Conclusions}

The main conclusions from our abundance analysis employing high-resolution optical spectra of HD 10613 and BD+04 2466 in order to obtain the abundance pattern and carbon isotopic ratio can be summarized as follows:

1. HD 10613 is another metal-poor barium star, not already shown as a binary system. However, its luminosity as well as its position in the diagrams showing abundance ratios versus metallicity give support to the interpretation that the observed overabundances of carbon and s-process elements in the photosphere of this star are due to mass transfer from a companion, formerly a TP-AGB star.

2. $\mathrm{BD}+04^{\circ} 2466$ is a $\mathrm{CH}$ star since its carbon-to-oxygen ratio is larger than unity $(\mathrm{C} / \mathrm{O}=3.63)$. In fact, $\mathrm{BD}+04^{\circ} 2466$ displays the main characteristics of the mass-transfer paradigm, i.e. presents overabundances of the elements created by the slow neutron capture reactions and carbon as well and has already been proved to be a member of a binary system (Jorissen et al. 2005). It was also shown that BD+04 2466 is another "lead star", since its $[\mathrm{Pb} / \mathrm{Ce}]$ ratio closely follows the theoretical predictions for a star at such metallicity. In fact, $\mathrm{BD}+04^{\circ} 2466$ lies in the same region of Fig. $10 \mathrm{occu}-$ pied by some $\mathrm{CH}$ stars that have already been classified as "lead stars".

Finally, it is interesting to note that BD $+04^{\circ} 2466$ is another star belonging to the LB91 short list of four stars categorized as "metal-deficient barium stars" that had its evolutionary status modified after the determination of the light element and lead abundances. Previous studies of other two stars from this list, HD 104340 and BD+03 2688 , showed that they are metal-poor AGB stars (Drake \& Pereira 2008; Jorissen et al. 2005).

Acknowledgements. This research has made use of the SIMBAD database, operated at CDS, Strasburg, France.

\section{References}

Allen, D. M., \& Barbuy, B. 2006, A\&A, 454, 895

Allende Prieto, C., Lambert, D. L., \& Asplund, M. 2001, ApJ, 556, L63 Alonso, A., Arribas, S., \& Martínez-Roger, C. 1999, A\&AS, 140, 261

Aoki, W., Ryan, S. G., Norris, J. E., et al. 2002, ApJ, 580, 1149

Barbuy, B., Jorissen, A., Rossi, S. C. F., \& Arnould, M. 1992, A\&A, 262, 216

Barbuy, B., Spite, M., Spite, F., et al. 2005, A\&A, 429, 1031

Bauschlicher, C. W., Langhoff, S. R., \& Taylor, P. R. 1988, ApJ, 332, 531

Bessell, M. S., Castelli, F., \& Plez, B. 1998, A\&A, 333, 231

Biemont, E., \& Godefroid, M. 1980, A\&A, 84, 361

Biemont, E., Grevesse, N., Hannaford, P., \& Lowe, R. M. 1981, ApJ, 248, 867

Bond, H. E. 1980, ApJS, 44, 517

Burbidge, E. M., Burbidge, G. R., Fowler, W. A., \& Hoyle, F. 1957, RvMP, 29, 547

Busso, M., Gallino, R., Lambert, D. L., Travaglio, C., \& Smith, V. V. 2001, ApJ, 557,802

Carbon, D. F., Barbuy, B., Kraft, R. P., et al. 1987, PASP, 99, 335

Carretta, E., Gratton, R., Cohen, J. G., Beers, T. C., \& Christlieb, N. 2002, AJ, 124,481

Catchpole, R. M., Robertson, B. S. C., \& Warren, P. R. 1977, MNRAS, 181, 391 Cayrel, R. 1988, Data Analysis, in The Impact of Very High S/N Spectroscopy on Stellar Physics, ed. G. Cayrel de Strobel, \& M. Spite (Dordrecht: Kluwer), 345

Clayton, D. D. 1988, MNRAS, 234, 1

Clegg, R. E. S., Tomkin, J., \& Lambert, D. L. 1981, ApJ, 250, 262

Davis, S. P., \& Phillips, J. G. 1963, The Red System of the CN molecule (Berkley and Los Angeles: Univ. of California Press), 214

del Peloso, E. F., Cunha, K., da Silva, L., \& Porto de Mello, G. F. 2005, A\&A, 441, 1149

Denissenkov, P. A., \& Pinsonneault, M. 2008, ApJ, 679, 1541

Depagne, E., Hill, V., Spite, M., et al. 2002, A\&A, 390, 187

Drake, J. J., \& Smith, G. 1991, MNRAS, 250, 89

Drake, N. A., \& Pereira, C. B. 2007, CNO and Li abundances in Barium-enriched stars, in Convection in Astrophysics, ed. F. Kupka, I. Roxburgh, \& K. Chan, 304

Drake, N. A., \& Pereira, C. B. 2008, AJ, 135, 1070

Dwivedi, P. H., Branch, D., Huffaker, J. N., \& Bell, R. A. 1978, ApJS, 36, 573

Edvardsson, B., Andersen, J., Gustafsson, B., et al. 1993, A\&A, 275, 101

François, P., Depagne, E., Hill, V., et al. 2003, A\&A, 403, 1105

Fulbright, J. P. 2002, AJ, 123, 404

Gómez, A. E., Luri, X., Grenier, S., et al. 1997, A\&A, 319, 881

Goriely, S., \& Mowlavi, N. 2000, A\&A, 362, 599

Gratton, R. G. 1989, A\&A, 208, 171

Gratton, R. G., \& Sneden, C. 1987, A\&A, 178, 179

Gratton, R. G., \& Sneden, C. 1988, A\&A, 204, 193

Gratton, R. G., \& Sneden, C. 1994, A\&A, 287, 927

Hartwick, F. D. A., \& Cowley, A. P. 1985, AJ, 90, 2244

Hannaford, P., Lowe, R. M., Grevesse, N., Biemont, E., \& Whaling, W. 1982, ApJ, 261, 736

Hill, V., Barbuy, B., Spite, M., et al. 2000, A\&A, 353, 557

Hobbs, L. M., Thorburn, J. A., \& Rebull, L. M. 1999, ApJ, 523, 797

Huber, R. P., \& Herzberg, G. 1979, Constants of Diatomic Molecules (New York: Van Nostrand) 
Iben, I., \& Renzini, A. 1983, ARA\&A, 21, 271

Jonsell, K., \& Edvardsson, B. 2005, A\&A, 440, 321

Jørgensen, U. G., Larsson, M., Iwamae, A., \& Yu, B. 1996, A\&A, 315, 204

Jorissen, A., Začs, L., Udry, S., Lindgren, H., \& Musaev, F. A. 2005, A\&A, 441 1135

Junqueira, S., \& Pereira, C. B. 2001, AJ, 122, 360

Kaufer, A., Stahl, O., Tubbesing, S., et al. 1999, The Messenger, 95, 8

Kovacs, I. 1969, Rotational Structure in the Spectra of Diatomic Molecules (Budapest: Akademiai Kiado)

Kurucz, R. L. 1993, CD-ROM 13, Atlas9 Stellar Atmosphere Programs and $2 \mathrm{~km} \mathrm{~s}^{-1}$ Grid (Cambridge: Smithsonian Astrophys. Obs)

Lambert, D. L. 1978, MNRAS, 182, 249

Lambert, D. L. 1981, in Physical Process in Red Giants, ed. I. Iben, \& A. Renzini (Dordrecht: D. Reidel Publ. Co.), 115

Lambert, D. L. 1994, Stellar photospheres and molecules - A view from the bridge, in Molecules in the Stellar Environment, IAU Coll., 146, 1

Lambert, D. L., \& Ries, L. M. 1981, ApJ, 248, 228

Lambert, D. L., Gustafsson, B., Eriksson, K., \& Hinkle, K. H. 1986, ApJS, 62 , 373

Lambert, D. L., Smith, V. V., \& Heath, J. 1993, PASP, 105, 568

Lambert, D. L., Heath, J. E., Lemke, M., \& Drake, J. 1996, ApJS, 103, 183

Lu, P. K. 1991, AJ, 101, 2229

Lucatello, S., Gratton, R., Cohen, J. G., et al. 2003, AJ, 125, 875

Luck, R. E., \& Bond, H. E. 1991, ApJS, 77, 515

MacConnell, D. J., Frye, R. L., \& Upgren, A. R. 1972, AJ, 77, 384

Martin, G. A., Fuhr, J. R., \& Wiese, W. L. 1988, J. Phys. Chem. Ref. Data, 17, 4

Masseron, T., van Eck, S., Famaey, B., et al. 2006, A\&A, 455, 1059

Mennessier, M. O., Luri, X., Figueras, F., et al. 1997, A\&A, 326, 722

Mishenina, T. V., \& Kovtyukh, V. V. 2001, A\&A, 370, 951

Pereira, C. B. 2005, AJ, 129, 2469

Pereira, C. B., \& Junqueira, S. 2003, A\&A, 402, 1061

Phillips, J. G., \& Davis, S. P. 1968, The Swan System of the $\mathrm{C}_{2}$ Molecule (Berkeley and Los Angeles: University of California Press)

Preston, G. W., \& Sneden, C. 2001, ApJ, 122, 1545

Reddy, B. E., Parthasarathy, M., Gonzalez, G., \& Bakker, E. J. 1997, A\&A, 328, 331

Reddy, B. E., Bakker, E. J., \& Hrivnak, B. J. 1999, ApJ, 524, 831

Reddy, B. E., Lambert, D. L., Gonzalez, G., \& Yong, D. 2002, ApJ, 564, 482
Reddy, B. E., Tomkin, J., Lambert, D. L., \& Allende Prieto, C. 2003, MNRAS, 340,304

Reyniers, M., Van Winckel, H., Biémont, E. \& Quinet, P. 2002, A\&A, 395, L35 Reyniers, M., Van Winckel, H., Gallino, R., \& Straniero, O. 2004, A\&A, 417, 269

Ruelas-Mayorga, A. 1997, RMxAA, 33, 9

Ryan, S. G., Norris, J. E., \& Bessell, M. S. 1991, AJ, 102, 303

Ryan, S. G., Norris, J. E., \& Beers, T. C. 1996, ApJ, 471, 254

Schadee, A. 1964, BAN, 17, 311

Shavrina, A. V., Polosukhina, N. S., Pavlenko, Ya. V., et al. 2003, A\&A, 409, 707

Sivarani, T., Bonifacio, P., Molaro, P., et al. 2004, A\&A, 413, 1073

Smith, V. V. 1984, A\&A, 132, 326

Smith, V. V. 1992, The barium stars, in Evolutionary Process in Interacting Binary Stars (Dordrecht: D. Reidel Publ. Co.), IAU Symp., 151, 103 Smith, V. V., \& Lambert, D. L. 1990, ApJS, 72, 387

Smith, G., Edvardsson, B., \& Frisk, U. 1986, A\&A, 165, 126

Smith, V. V., Cunha, K., Jorissen, A., \& Boffin, H. M. J. 1996, A\&A, 315, 179

Smith, V. V., Lambert, D. L., \& Nissen, P. E. 1998, ApJ, 506, 405

Sneden, C. 1973, Ph.D. Thesis, Univ. of Texas

Sneden, C. 1983, PASP, 95, 745

Sneden, C., \& Lambert, D. L. 1982, ApJ, 259, 381

Sneden, C., Lambert, D. L., \& Pilachowski, C. A. 1981, ApJ, 247, 1052

Sneden, C., Gratton, R. G., \& Crocker, D. A. 1991, A\&A, 246, 354

Sneden, C., McWilliam, A., Preston, G. W., et al. 1996, ApJ, 467, 819

Stancliffe, R. J., Glebbeek, E., Izzard, R. G., \& Pols, O. R. 2007, A\&A, 464, L57

Thompson, I. B., Ivans, I. I., Bisterzo, S., et al. 2008, ApJ, 677, 556

Tomkin, J., \& Lambert, D. L. 1984, ApJ, 279, 220

van Winckel, H., \& Reyniers, M. 2000, A\&A, 354, 135

Van Eck, S., Goriely, S., Jorissen, A., \& Plez, B. 2003, A\&A, 404, 291

Vanture, A. 1992a, AJ, 103, 2035

Vanture, A. 1992b, AJ, 104, 1986

Vanture, A. 1992c, AJ, 104, 1997

Wallerstein, G. 1997, RvMP, 69, 995

Wiese, W. L., \& Martin, G. A. 1980, NSDRS-NBS, 68

Wiese, W. L., Smith, M. W., \& Miles, B. M. 1969, NBS Ref. Data. Ser.

Wyller, A. A. 1966, ApJ, 143, 828

Začs, L., Klochkova, V. G., \& Panchuk, V. E. 1995, MNRAS, 275, 764 